\title{
Determination of tube theory parameters using a simple grid model as an example
}

Article

Accepted Version

Likhtman, A., Talib, M., Vorselaars, B. and Ramirez, J. (2013)

Determination of tube theory parameters using a simple grid model as an example. Macromolecules, 46 (3). pp. 1187-1200. ISSN 0024-9297 doi: https://doi.org/10.1021/ma302103p Available at https://centaur.reading.ac.uk/31285/

It is advisable to refer to the publisher's version if you intend to cite from the work. See Guidance on citing.

To link to this article DOI: http://dx.doi.org/10.1021/ma302103p

Publisher: American Chemical Society

All outputs in CentAUR are protected by Intellectual Property Rights law, including copyright law. Copyright and IPR is retained by the creators or other copyright holders. Terms and conditions for use of this material are defined in the End User Agreement.

\section{www.reading.ac.uk/centaur}

\section{CentAUR}

Central Archive at the University of Reading

Reading's research outputs online 


\title{
Determination of tube theory parameters using a simple grid model as an example
}

\author{
Alexei E. Likhtman, ${ }^{* \dagger}$ Mohamad S. Talib, ${ }^{*, \dagger, \ddagger}$ Bart Vorselaars, ${ }^{*}, \dagger, \mathbb{I l}$ and Jorge \\ Ramirez $z^{*} \dagger, \S$
}

School of Mathematical and Physical Sciences, University of Reading, Reading RG6 6AX, UK

\begin{abstract}
Although the tube theory is successful in describing entangled polymers qualitatively, a more quantitative description requires precise and consistent definitions of its parameters. Here we investigate the simplest model of entangled polymers, namely a single Rouse chain in a cubic lattice of line obstacles, and illustrate the typical problems and uncertainties of the tube theory. In particular we show that in general one needs 3 entanglement related parameters, but only 2 combinations of them are relevant for the long-time dynamics. Conversely, the plateau modulus can not be determined from these two parameters and requires a more detailed model of entanglements with explicit entanglement forces, such as the slipsprings model. It is shown that for the grid model the Rouse time within the tube is larger than the Rouse time of the free chain, in contrast to what the standard tube theory assumes.
\end{abstract}

\section{Introduction}

Dynamics of entangled polymers is one of the most intriguing unsolved problems of modern polymer science, which has a relatively simple formulation and yet immense industrial importance through its direct relevance to polymer processing. The dynamics of short chains can be qualitatively described by the Rouse model.

\footnotetext{
*To whom correspondence should be addressed: A.Likhtman@ reading.ac.uk

${ }^{\dagger}$ School of Mathematical and Physical Sciences, University of Reading, Reading RG6 6AX, UK

†Present address: Faculty of Computer Science and Information System, Universiti Teknologi Malaysia, 81310 UTM Skudai, Johor, Malaysia

IPresent address: Department of Physics, University of Warwick, Coventry CV4 7AL, UK

${ }^{\S}$ Present address: Chemical Engineering Department, Technical University of Madrid, Jose Gutierrez Abascal 2, 28006 Madrid, Spain
} 
As chains get longer, the stress relaxation slows down significantly and develops a stress plateau at the level $G_{N}^{(0)}$, and finally decays to zero at long time $\tau_{d} \sim M^{3.4}$ where $M$ is the polymer molecular weight. Usually a concept of tube effectively surrounding each chain is used to describe long well entangled polymers. It is successful and elegant concept which explains many scaling power laws simultaneously with a limited set of simple assumptions. In particular, it managed to explain why the viscosity of linear chains grows with molecular weight as $\eta \sim M^{3.4}$ whereas for star polymers $\eta \sim \exp (\alpha M)$.

Such success drove a large scale effort to make the tube theory quantitative, ${ }^{1}$ which led to some significant achievements. ${ }^{2}$ However, the modern tube theory became much more complicated than the original intuitive picture. Most importantly, it contains many contradicting parts and unverified assumptions, and different pieces of the tube theory developed by different research groups can not be reconciled against each other. We think that the origin of such situation lies in the most basic postulates of the tube theory, and in particular in the fact that there is no clear constructive definition of the tube, which makes the verification of the assumptions problematic.

One of the problems of the tube theory is the determination of the main parameter of the theory, namely the number of Kuhn segments between entanglements $N_{e}$ or the so-called tube diameter $a=\sqrt{N_{e}} b$, where $b$ is the statistical segment of the chain. In the recent years primitive path analysis (PPA) gained much attention ${ }^{3-5}$ as a quick method to predict $N_{e}$ from the shortest path between the chain ends which preserves the topology of the chains. The PPA method is based on the assumption that the chains are effectively diffusing along the shortest path available to them, which seems like a lower bound rather than an exact statement. The only verification provided to PPA is the comparison of its predictions for the plateau modulus $G_{N}^{(0)}$, measured in simulations or in experiment. The scaling dependence on the chain stiffness is captured, but the numerical precision of the prediction is not clear. Besides, the plateau modulus is not the only quantity one needs to know to provide the tube theory predictions (see below).

In this paper we consider a simple model of a single entangled chain in an array of line obstacles and study its static and dynamics properties in great details. Due to the simplicity of the model, we are able to obtain accurate data for sufficiently long chains and study several ways of obtaining the tube parameters, as well as test several basic assumptions of the tube theory. We shall use this simple model to develop techniques and definitions which can then be applied to more complex systems such as molecular dynamics simulations of polymer melts. In section 2 we shall describe the model and present the standard "experimental" observables. We will also show that the conventional PPA and tube axis methods give very bad predictions for the plateau modulus and $N_{e}$ of this model. In section 3 we reconsider the tube theory assumptions and derive more 
general expressions for the monomer mean-square displacement. In section 4 the results from the static analysis of chain configurations will be presented, whereas in section 5 we will extract the tube parameters from dynamic observables. We conclude with a discussion and outlook in section 6.

\section{Model and main observables}

We will use one of the simplest models for entanglements to illustrate our ideas about the tube theory. Namely, we shall consider a single Rouse chain in a cubic mesh of line obstacles; hereafter it will be called the grid model. The Rouse chain is a chain of massless beads with frictions $\zeta$, connected by linear springs with spring constant $k=3 k_{B} T / b^{2}$ where $k_{B}$ is the Boltzmann constant, $T$ the absolute temperature and $b_{3 \mathrm{D}}$ the statistical segment of the chain. The subscript 3D is used here to distinguish this case from the onedimensional Rouse model parameters introduced later. The equations of motion for the position $\mathbf{R}_{i}$ of the $i$ 'th bead and end beads are

$$
\begin{aligned}
\zeta \frac{d \mathbf{R}_{0}}{d t} & =\frac{3 k_{B} T}{b_{3 \mathrm{D}}^{2}}\left(\mathbf{R}_{1}-\mathbf{R}_{0}\right)+\mathbf{f}_{0}(t) \\
\zeta \frac{d \mathbf{R}_{i}}{d t} & =\frac{3 k_{B} T}{b_{3 \mathrm{D}}^{2}}\left(\mathbf{R}_{i+1}+\mathbf{R}_{i-1}-2 \mathbf{R}_{i}\right)+\mathbf{f}_{i}(t), \quad 1 \leq i \leq N-1 \\
\zeta \frac{d \mathbf{R}_{N}}{d t} & =\frac{3 k_{B} T}{b_{3 \mathrm{D}}^{2}}\left(\mathbf{R}_{N-1}-\mathbf{R}_{N}\right)+\mathbf{f}_{N}(t)
\end{aligned}
$$

where $N$ is the number of springs, and $\mathbf{f}_{i}(t)$ is the random force on a bead number $i$ at time $t$, which is assumed to be uncorrelated with the forces on other beads or at different moments of time. According to the fluctuation-dissipation theorem, the random forces should satisfy

$$
\left\langle f_{i \alpha}(t) f_{j \beta}\left(t^{\prime}\right)\right\rangle=2 k_{B} T \zeta \delta_{i j} \delta_{\alpha \beta} \delta\left(t-t^{\prime}\right)
$$

where the Greek indices denote Cartesian components, $\delta_{i j}=1$ if $i=j$ and $\delta_{i j}=0$ otherwise, and $\delta(\cdots)$ denotes the Dirac delta function.

The entanglements are modelled by a set of straight lines creating a simple cubic lattice with grid spacing $g$, which is the only entanglement-related parameter of the model. Each line is oriented parallel to one of the Cartesian coordinate axes. To mimic entangled dynamics, we reject all monomer motions that would result in polymer bonds crossing any of the lattice lines. We simulated these equations using the predictor-corrector scheme, following the algorithm below 
1. Generate a list of random permutation of monomer indices $[0,1, \ldots, N-1, N]$

2. For each bead $i$ from the list do:

3. Calculate the regular force from the neighboring beads on bead $i$

$$
\mathbf{f}_{i}^{(p)}:=\frac{3 k_{B} T}{b_{3 \mathrm{D}}^{2}}\left(\mathbf{R}_{i+1}+\mathbf{R}_{i-1}-2 \mathbf{R}_{i}\right) .
$$

Obviously, if $i=0$ or $i=N$ then the equation for chain ends is used instead (see eq. 1).

4. Generate the predicted position of bead $i$ using the regular and the random forces

$$
\mathbf{R}_{i}^{(p)}:=\mathbf{R}_{i}+\frac{1}{\zeta} \mathbf{f}_{i}^{(p)} \Delta t+\sqrt{\frac{2 k_{B} T}{\zeta} \Delta t} \xi_{i}
$$

where $\Delta t$ is the time step and $\xi_{i}$ is a vector containing three normally distributed random numbers each having zero average and unit variance.

5. Calculate the corrected force, which is the force at the predicted position

$$
\mathbf{f}_{i}^{(c)}:=\frac{3 k_{B} T}{b_{3 \mathrm{D}}^{2}}\left(\mathbf{R}_{i+1}+\mathbf{R}_{i-1}-2 \mathbf{R}_{i}^{(p)}\right)
$$

6. Calculate the corrected position

$$
\mathbf{R}_{i}^{(c)}:=\mathbf{R}_{i}^{(p)}+\frac{\Delta t}{2 \zeta}\left(\mathbf{f}_{i}^{(c)}-\mathbf{f}_{i}^{(p)}\right)
$$

7. Verify if moving the bead from $\mathbf{R}_{i}$ to $\mathbf{R}_{i}^{(c)}$ resulted in bonds connecting bead $i$ to beads $i-1$ and $i+1$ crossing a line obstacle. If it did not, assign

$$
\mathbf{R}_{i}:=\mathbf{R}_{i}^{(c)}
$$

The check has been carried out as follows. The vectors $\mathbf{R}_{i}, \mathbf{R}_{i}^{(c)}$ and either $\mathbf{R}_{i-1}$ or $\mathbf{R}_{i+1}$ form a triangle. For both triangles we check if any violation occurs. If a triangle is entirely within a cell, there are obviously no constraints violated. Otherwise we determine all the cells any of the sides of the triangle passes through, and explicitly determine if any of the line obstacles of these cells intersects 
the triangle using an algorithm similar to Refs. ${ }^{6,7}$ For the primitive path construction (to be discussed later) we will use a slightly different variant. ${ }^{8}$

These steps are repeated $N+1$ times for each chain each time step, so that every bead is attempted to move once. Since we have a freedom to choose the units of time, space and energy arbitrarily, we set $b_{3 \mathrm{D}}=1$, $\zeta=1$ and $k_{B} T=1$, which makes the longest relaxation of the chain without entanglements, or the Rouse time, equal to

$$
\tau_{R} \approx \frac{b_{3 \mathrm{D}}^{2} \zeta(N+1)^{2}}{3 \pi^{2} k_{B} T}=\frac{(N+1)^{2}}{3 \pi^{2}}
$$

Likewise, we can define a natural time unit, independent of chain length, $\tau_{0}$, as

$$
\tau_{0} \equiv \frac{\tau_{R}}{(N+1)^{2}}=\frac{1}{3 \pi^{2}}
$$

The main results from the grid model for the middle monomer mean-square displacement $g_{1 \text {,mid }}(t)$, end-toend vector autocorrelation function $\Phi(t)$ (normalized such that $\Phi(0)=1$ ) and stress auto-correlation function $G(t)$ are shown in Fig. 1 for different grid sizes $g=1,2,4$ and different chain lengths. At early time the results follow the Rouse model predictions (especially for larger grids), which means that the obstacles do not play a significant role on small time scale.

The fraction of rejected moves was about $2.3 \sqrt{\Delta t / g}$, so for large grids and for the timestep we used $(\Delta t=0.01)$ it should not have any significant effect on the fast dynamics. On larger timescales the chains have to move predominantly parallel to themselves, and therefore show dynamics slower than the Rouse chains: the stress relaxation develops a plateau, and the mean square monomer displacement a characteristic $t^{1 / 4}$ behaviour predicted by Doi and Edwards. ${ }^{9}$ For long chains the longest relaxation times become proportional to $N^{3.4}$ as observed experimentally (Fig. 2), and the deviation from the Rouse predictions happen at larger $N$ for larger grids. The longest relaxation time was extracted by fitting $G(t)$ and $\Phi(t)$ with a sum of exponential modes (or Maxwell modes) using the Reptate software package. ${ }^{10}$

The static properties of the chains are unaffected by the obstacles since their volume is infinitely small. This is illustrated in Fig. 1 (a), where we plotted the average inter-monomer distances defined as

$$
d(s)=\frac{1}{N-s+1} \sum_{i=0}^{N-s} \frac{\left\langle\left(\mathbf{R}_{i+s}-\mathbf{R}_{i}\right)^{2}\right\rangle}{s}
$$

All results are in agreement with the Rouse model predictions $d(s)=1$ within the statistical noise and small deviations due to the numerical integration error (less than $1 \%$ ). 

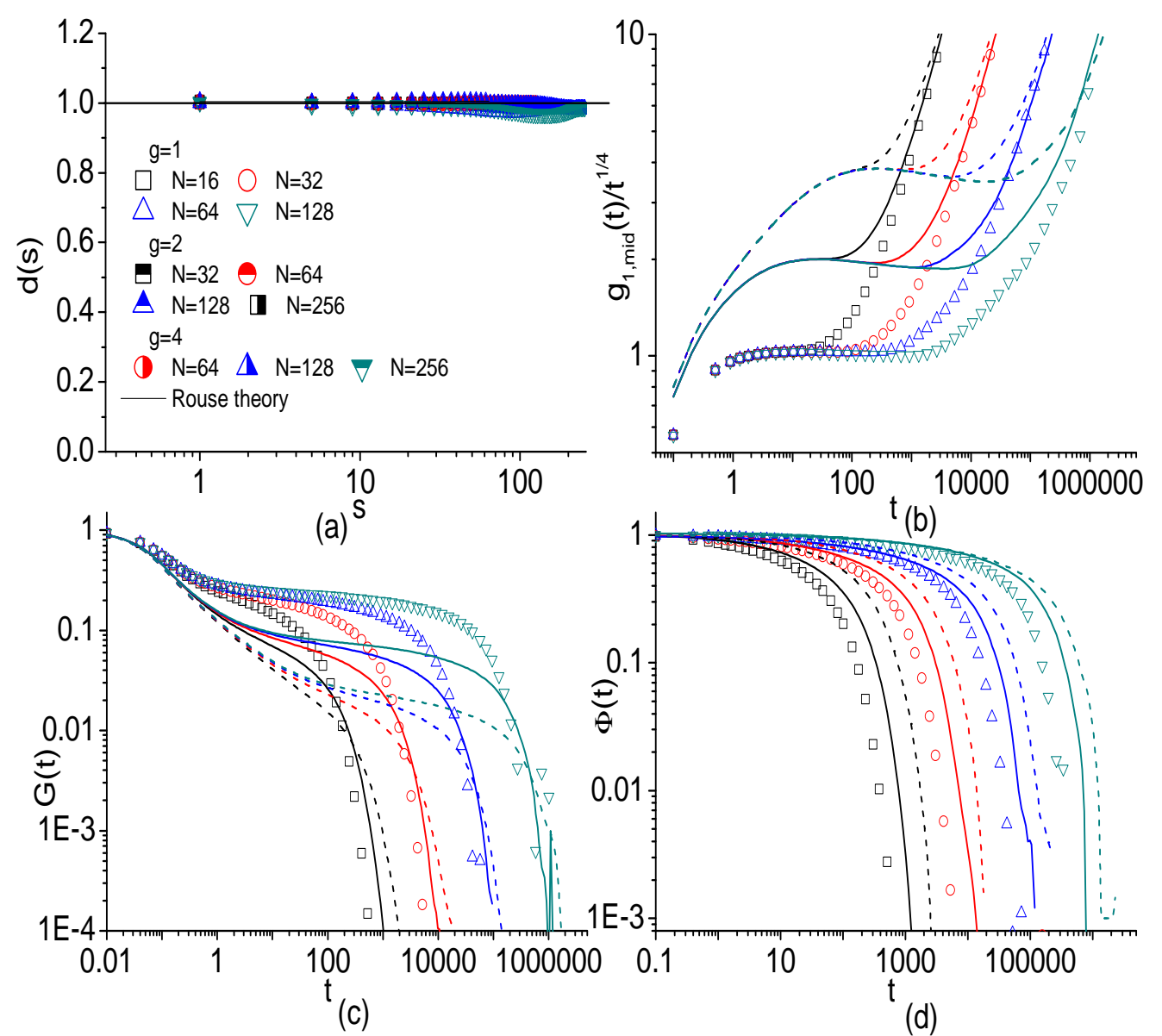

Figure 1: Main results for the grid model. Symbols correspond to $g=1$ with $N=16,32,64,128$, lines for $g=2$ with $N=32,64,128,256$ and the dashed lines for $g=4$ with $N=64,128,256,512$.

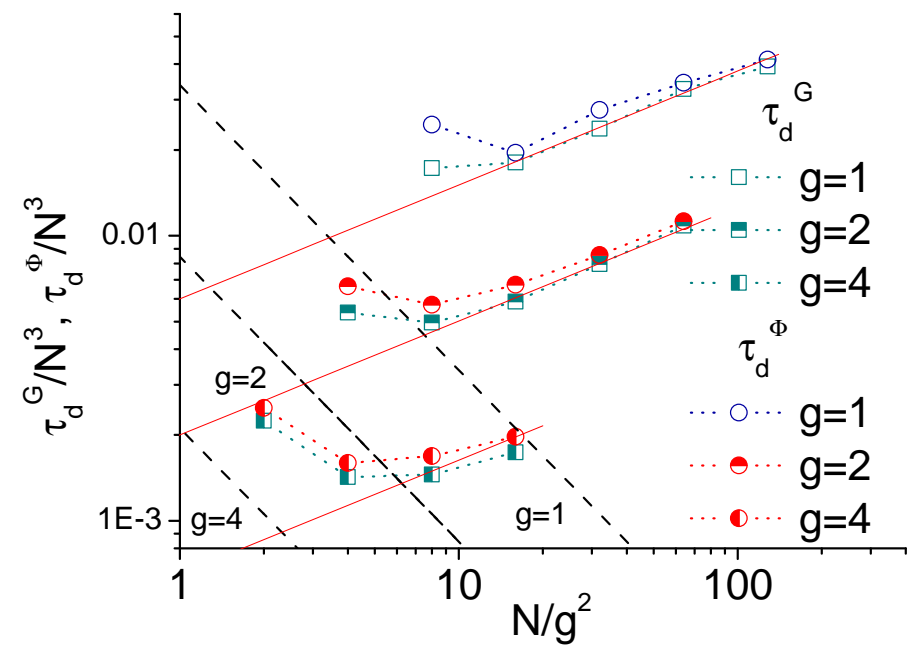

Figure 2: Normalized longest relaxation time of $G(t)$ and $\Phi(t)$ for different grid sizes and chain lengths. The dashed lines are the $\tau_{R} / N^{3}$ for each grid size. Slope 0.4 is shown by the solid lines. 
One can see that qualitatively the model reproduces all tube model predictions. It also has an advantage that for small times it follows the unentangled Rouse dynamics, whereas the tube model only gives the correct predictions after times $t \gg \tau_{e}=N_{e}^{2} \tau_{0}$. The main question of this paper is: can this model be described quantitatively by the tube model, and if so, how to obtain the tube model parameters required for such quantitative description.

\section{Tube theory}

In this section we re-derive the main quantitative predictions of the tube theory, paying special attention to the assumptions and involved approximations. The tube theory of Doi and Edwards is based on two basic assumptions:

1. There exists a path with the measurable length $L$, and the chain motion can be projected onto it. In other words, at every moment of time one can specify each monomer's one-dimensional position along this path. On large length-scales this path should follow the chain and therefore should behave as a random walk. One can not specify small scale statistics of this path without additional approximations.

2. The projected dynamics of the chain along this path is the same as that of the free one-dimensional Rouse chain stretched by the ends. The stretching force is set to maintain the average path length equal to $L$.

These simple assumptions are sufficient to calculate the mean square displacement (MSD) of the middle monomer. We start from the MSD along the tube. Let's denote it as $g_{s}(t) \equiv\left\langle(s(t)-s(0))^{2}\right\rangle$, where $s(t)$ is the one-dimensional position of the middle monomer along the tube. For a Rouse chain, the displacement is not affected by a constant stretching force, and therefore we can use the results for a free Rouse chain ${ }^{11}$

$$
g_{s}(t)=\frac{2 k_{B} T}{(N+1) \zeta_{1 \mathrm{D}}}\left(t+2 \sum_{p=1}^{N / 2} \tau_{2 p}\left[1-\exp \left(-\frac{t}{\tau_{2 p}}\right)\right]\right) ; \quad \tau_{p}=\frac{\zeta_{1 \mathrm{D}} b_{1 \mathrm{D}}^{2}}{12 k_{B} T} \sin ^{-2}\left(\frac{\pi p}{2(N+1)}\right)
$$

where $\tau_{p}$ is the relaxation time of the Rouse mode number $p$. Note that only even Rouse modes contribute to the middle monomer displacement. Apart from the temperature, this result depends on the friction $\zeta_{1 D}$ and statistical segment length $b_{1 \mathrm{D}}$, which we mark with the subscript $1 \mathrm{D}$ to stress that the friction and the effective spring constant along the one-dimensional tube path $k=\frac{3 k_{B} T}{b_{1 \mathrm{D}}^{2}}$ might be different from the free Rouse chain parameters in three dimensions. In our notations the unstretched Rouse chain in one dimension will 
have average squared end-to-end vector:

$$
\left\langle R_{e, 1 \mathrm{D}}^{2}\right\rangle=\frac{N b_{1 \mathrm{D}}^{2}}{3}
$$

Eq. 4 contains three time domains separated by two characteristic times: $\tau_{R}^{1 \mathrm{D}}=\tau_{1}$ is the relaxation time of the slowest Rouse mode in one dimension, called the Rouse time, and $\tau_{0}^{1 \mathrm{D}} \equiv \tau_{R}^{1 \mathrm{D}} /(N+1)^{2}$ is the standard notation for the shortest relaxation time (note, however, that the actual fastest relaxation time $\tau_{N} \approx \frac{\zeta b^{2}}{12 k_{B} T}$ is about 2.5 times larger than $\tau_{0}=\frac{\zeta b^{2}}{3 \pi^{2} k_{B} T}$, eq. 2$)$. In these regimes eq. 4 can be simplified as

$$
g_{s}(t) \approx \frac{2 k_{B} T}{\zeta_{1 \mathrm{D}}}\left\{\begin{array}{cc}
t, & t \ll \tau_{0}^{1 \mathrm{D}} \\
b_{1 \mathrm{D}} \sqrt{\frac{t \zeta_{1 \mathrm{D}}}{3 \pi k_{B} T}}, & \tau_{0}^{1 \mathrm{D}} \ll t \ll \tau_{R}^{1 \mathrm{D}} \\
\frac{t}{(N+1)}, & t \gg \tau_{R}^{1 \mathrm{D}}
\end{array}\right.
$$

The first and third regimes correspond to the single bead and the center of mass diffusion in one dimension, whereas the second regime shows the famous subdiffusive behaviour.

Eq. 4 can be approximated for $t \gg \tau_{0}^{1 \mathrm{D}}$ by

$$
g_{s}(t) \approx \frac{2 N b_{1 \mathrm{D}}^{2}}{3 \pi^{2}} \sqrt{\frac{t}{\tau_{R}^{1 \mathrm{D}}}}\left(\left(\frac{t}{\tau_{R}^{1 \mathrm{D}}}\right)^{3 / 2}+\pi^{3 / 2}\right)^{1 / 3}
$$

Now we define the main parameter $a$ describing the tube statistics as follows: assume the tube path can be represented as a freely-jointed chain with $Z$ steps of length $a$. Since the end-to-end vector of the tube and the chain should coincide for long enough chains, we have two equations

$$
\begin{aligned}
L & =Z a \\
R_{e}^{2} & =Z a^{2}=N b_{3 \mathrm{D}}^{2}
\end{aligned}
$$

This means that once the tube length is defined (see the first assumption of the tube theory), we can define $a$ and $Z$ as

$$
\begin{aligned}
a & \equiv \frac{N b_{3 \mathrm{D}}^{2}}{L} \\
Z & =\frac{L^{2}}{N b_{3 \mathrm{D}}^{2}}
\end{aligned}
$$


It is clear from these definitions that $a$ should be called the tube Kuhn step, i.e., a step length of an equivalent freely-jointed chain with the same contour length and the same end-to-end distance, and $Z$ is the number of these Kuhn segments in the chain. This $Z$ is not necessarily the number of entanglements since entanglements are usually associated with the number of turns of the tube path (see Appendix A for a detailed discussion of the differences). If the tube path does not have the freely-jointed chain statistics (independent steps of equal length), then the number of steps will be in general different from $Z$. We also note that if we use definitions eq. 8 , we should avoid calling $a$ the tube diameter, since it clearly characterizes the tube properties along the tube path, not perpendicular to it. Below we will sometimes call "tube Kuhn step" by the shorter name "tube step".

Using this definition, we deduce that two tube segments with separation $\Delta s$ along the tube have the following mean square distance in real space

$$
\left\langle\Delta r^{2}\right\rangle=|\Delta s| a
$$

To calculate the middle monomer mean square displacement in real space $g_{1, \text { mid }}(t)$ we start from the probability to observe the displacement $\Delta s$ along the tube, which is Gaussian with zero mean, thus

$$
P_{s}\left(\Delta s, g_{s}(t)\right)=\frac{1}{\sqrt{2 \pi g_{s}(t)}} \exp \left(-\frac{\Delta s^{2}}{2 g_{s}(t)}\right)
$$

If two tube segments are separated by the tube length $\Delta s$, their separation in real space $r$ is distributed as

$$
P_{r}(r, \Delta s)=\left(\frac{3}{2 \pi|\Delta s| a}\right)^{3 / 2} 4 \pi r^{2} \exp \left(\frac{-3 r^{2}}{2|\Delta s| a}\right)
$$

i.e., standard spherically symmetric Gaussian with $\left\langle r^{2}\right\rangle=|\Delta s| a$ (eq. 9).

Thus, the mean square displacement in real space is

$$
g_{1, \text { mid }}(t)=\int_{-\infty}^{\infty} d \Delta s P_{s}\left(\Delta s, g_{s}(t)\right) \int_{0}^{\infty} d r r^{2} P_{r}(r, \Delta s)
$$

The inner integral over $r$ gives $|\Delta s| a$, and the outer integral then gives

$$
g_{1, \text { mid }}(t)=2 a \int_{0}^{\infty} d \Delta s \Delta s P_{s}\left(\Delta s, g_{s}(t)\right)=a \sqrt{\frac{2}{\pi} g_{s}(t)}
$$


This is a factor of $\sqrt{2 / \pi} \approx 0.798$ smaller than was sometimes assumed in the tube theory, ${ }^{12}$ where instead of integrating over $\Delta s$ the most likely value $\sqrt{g_{s}(t)}$ was assumed. Note however that in the book of Doi and Edwards $^{9}$ (eq. 6.38) correct calculations are reported, giving exactly the same results as eq. 11 .

Combining eqs. 4 and 11 leads to

$$
\begin{aligned}
g_{1, \text { mid }}(t) & =a \sqrt{\frac{4 k_{B} T}{\pi(N+1) \zeta_{1 \mathrm{D}}}\left(t+2 \sum_{p=1}^{N / 2} \tau_{2 p}\left[1-\exp \left(-\frac{t}{\tau_{2 p}}\right)\right]\right)} \\
& \approx a b_{1 \mathrm{D}} \sqrt{N} \frac{2}{3^{1 / 2} \pi^{3 / 2}} \begin{cases}\left(\frac{\pi t}{\tau_{R}^{1 \mathrm{D}}}\right)^{1 / 4}, & \tau_{0}^{1 \mathrm{D}} \ll t \ll \tau_{R}^{1 \mathrm{D}} \\
\left(\frac{t}{\tau_{R}^{1 \mathrm{D}}}\right)^{1 / 2}, & \tau_{R}^{1 \mathrm{D}} \ll t \ll \tau_{d}^{1 \mathrm{D}}\end{cases}
\end{aligned}
$$

The second part of this equation is written in a form that stresses that the MSD around the Rouse time depends only on two combinations: $a b_{1 \mathrm{D}} \sqrt{N}$ and $\tau_{R}^{1 \mathrm{D}}$, out of the 5 model parameters $\left(a, b_{1 D}, \xi_{1 D}, N, k_{B} T\right)$.

An important note is due here: the above derivation for eq. 13 does not require the assumption that the tube is a freely-jointed path of equal segments $a$. Any other statistics will lead to exactly the same result for long enough chains. Only the rate of convergence to this result as $N \rightarrow \infty$ will depend on the exact tube statistics. This is in contrast to the plateau modulus prediction ${ }^{9}$

$$
G_{N}^{(0)}=\frac{4}{5} c \frac{k_{B} T}{N_{e}}
$$

where $c$ is the monomer number density and $N_{e}$, the so-called number of monomers between entanglements, is defined as

$$
N_{e}=N / Z=a^{2} / b_{3 \mathrm{D}}^{2}
$$

To derive eqs. 14 and 15 a lot of assumptions about the tube on the length scales of $a$ are required, which are not even specified explicitly.

Equation 13 can be complemented by an early time free Rouse behaviour, which is not affected by entanglements

$$
g_{1, \text { mid }}(t)=\frac{6 k_{B} T}{\zeta_{3 \mathrm{D}}}\left\{\begin{array}{cc}
t, & t \ll \tau_{0}^{3 \mathrm{D}} \\
b_{3 \mathrm{D}} \sqrt{\frac{t \zeta_{3 \mathrm{D}}}{3 \pi k_{B} T}}, & \tau_{0}^{3 \mathrm{D}} \ll t \ll \tau_{e}
\end{array}\right.
$$

analogous to eq. 4 . The transition between eqs. 16 and 13 is not described by any theory: the tube theory in present form works only at much longer timescales, and the Rouse theory obviously fails when entanglements start to play a role. We denote the transition time by $\tau_{e}$ - the time when entanglements start to play a role and the dynamics deviate from the Rouse model predictions. 


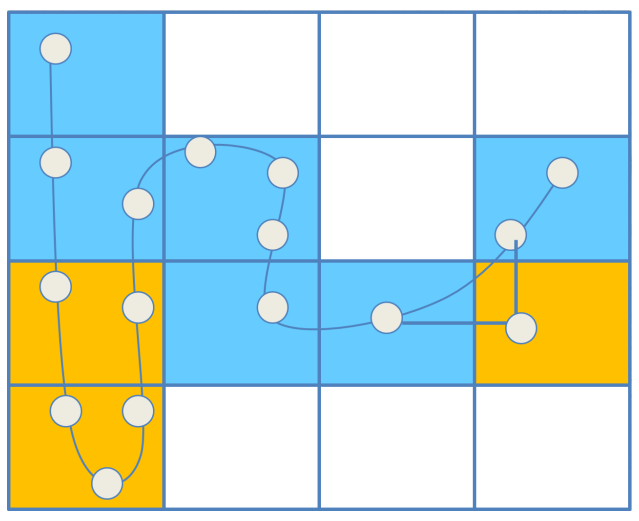

Figure 3: Tube construction steps illustrated on a simple two-dimensional example.

\section{Static analysis}

In order to obtain the tube parameters, one can use static analysis by generating many independent chain configurations. It is an easy task in our model since the chain statistics are exactly the same as for the free Rouse chain, namely Gaussian distributed bond distances. For each configuration, we measure the tube length by two different constructions: the tube axis and the primitive path.

In the tube axis method, used previously in lattice models analytically and numerically, ${ }^{13,14}$ we construct the tube by connecting the centers of the cubes belonging to the tube. To find all the cubes belonging to the tube, we follow the tube construction described in ref. ${ }^{14}$ We first mark all cubes which the chain crosses (either having a bead or a bond passing through), deleting repeating segments so that neighbouring cubes should be different. Then, we remove all the cubes belonging to unentangled loops by a simple algorithm: if the tube segments $i$ and $i+2$ (two apart) are in the same cell, cubes $i+1$ and $i+2$ are deleted. This procedure is repeated until there are no more cubes satisfying this condition. The tube length is then just $\left(Z_{c}-1\right) g$ where $Z_{c}$ is the number of cubes in the tube. The tube construction is illustrated graphically in Fig. 3.

The primitive path is the shortest distance between the chain ends, which can be obtained by moving the chain beads without crossing any grid lines. It can be constructed by fixing the chain ends and allowing other beads to move, slowly reducing the temperature. To make this procedure more effective, some additional moves along the grid lines, which reduce the chain length, can be introduced. In the context of molecular dynamics, primitive path algorithms differ from each other in details since all chains are pulled taut simultaneously. For our simple single chain model, however, there is only one unique solution, and all literature algorithms give equivalent results.

As a result of these two procedures, we obtain the probability distribution of the tube length using two 


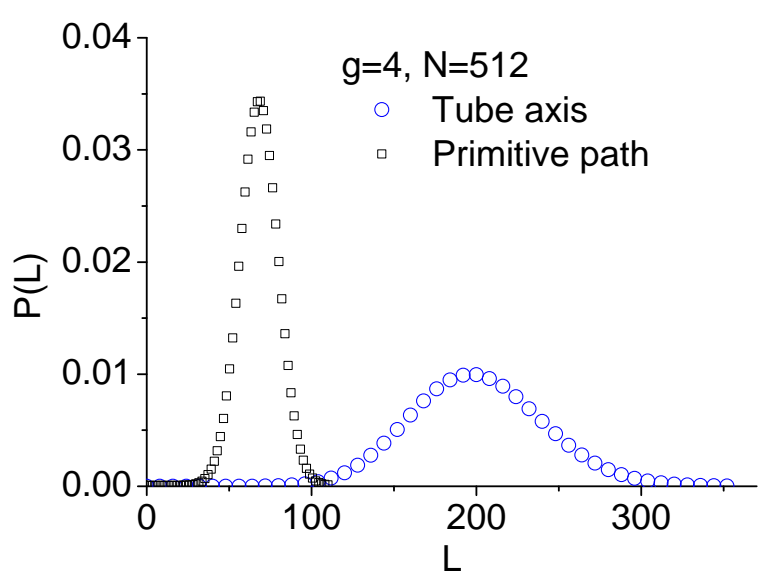

Figure 4: Probability distribution of tube axis and primitive path lengths for $N=512$ and $g=4$. It is clear that the primitive path is shorter and has smaller variance than the tube axis.

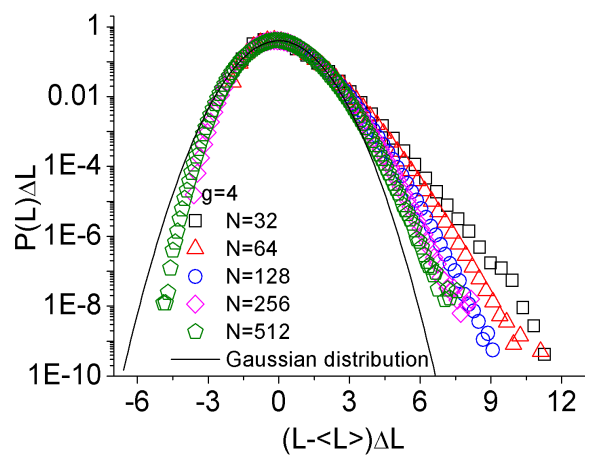

(a)

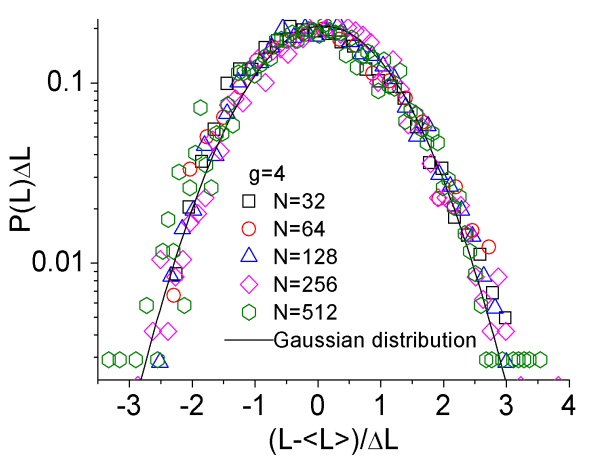

(b)

Figure 5: Probability distribution of (a) tube axis and (b) primitive path lengths for $g=4$ with various chain lengths. 


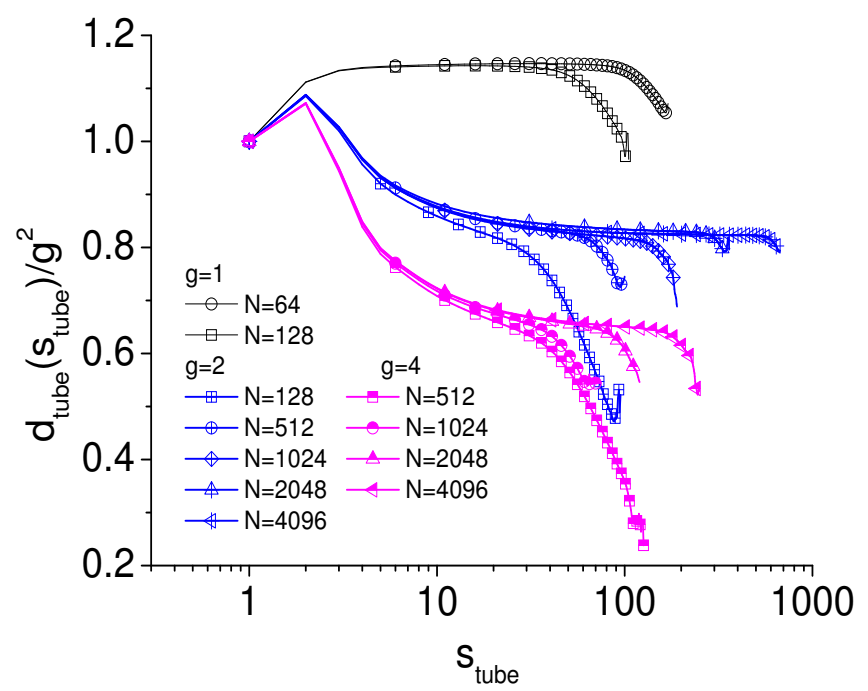

Figure 6: Mean square spatial distance of the tube axis normalised by $g^{2}$.

tube definitions: $P_{t a}(L)$ from the tube axis and $P_{p p}(L)$ from the primitive path. An example of such distributions is shown in Fig. 4 for $g=4$ and $N=512$. If the partial tube segments at the end are neglected the primitive path length is by definition always shorter than the tube axis length. The tube axis method is computationally much cheaper and therefore better statistics can be obtained. Fig. 5(a) shows that $P_{t a}(L)$ deviates from the standard Gaussian form for $L \ll\langle L\rangle$ and $L \gg\langle L\rangle$, however these deviations are only visible for very improbable lengths. It is clear that these deviations are not important for dynamics of long enough linear chains, whereas for branched chains $P_{t a}(0)$ plays an essential role in predicting the arm retraction time. ${ }^{1}$ Thus, for the purposes of this paper, $P(L)$ can be characterized by the average length and its variance

$$
\begin{aligned}
\langle L\rangle & =\int_{0}^{\infty} L P(L) d L \\
\left\langle\Delta L^{2}\right\rangle & =\int_{0}^{\infty}(L-\langle L\rangle)^{2} P(L) d L
\end{aligned}
$$

Fig. 5 shows $P(L)$ from two methods in a normalized form, where the averages are subtracted and the horizontal axis is rescaled by $\Delta L=\sqrt{\left\langle\Delta L^{2}\right\rangle}$. The solid line shows the Gaussian distribution with zero mean and unit variance. It is clear that as $N \rightarrow \infty$, the $P_{t a}(L)$ approaches a Gaussian distribution. The statistics for the primitive path is worse and therefore does not reveal any statistically significant deviations from the Gaussian distribution.

Apart from the total tube length, we can get more detailed information about the tube statistics by constructing a function $d_{\text {tube }}\left(s_{\text {tube }}\right)$ similar to eq. 3 but for the tube vertices. In this case, $s_{\text {tube }}$ will have the 


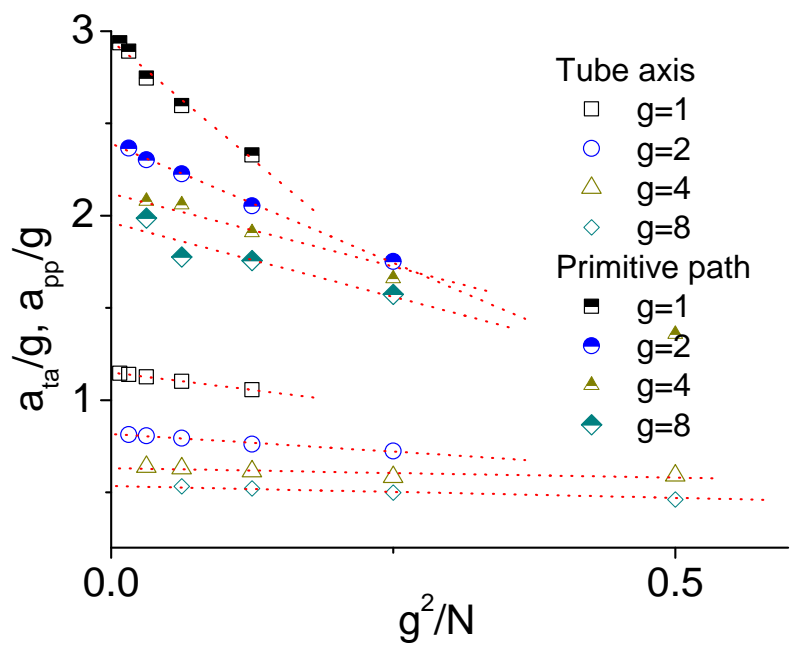

Figure 7: Tube Kuhn step extracted from the tube axis $\left(a_{t a}\right)$ and the primitive path $\left(a_{p p}\right)$ constructions, illustrating extrapolation procedure to $N \rightarrow \infty$.

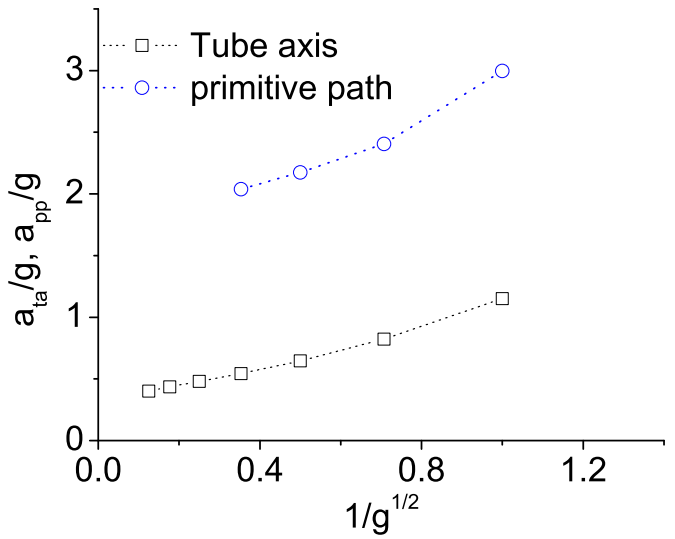

Figure 8: Extrapolated values of tube Kuhn step normalized by the grid size. 
meaning of the distance along the tube. Fig.6 shows tube $d_{\text {tube }}\left(s_{\text {tube }}\right)$ for different grids. All curves start with $d_{\text {tube }}\left(s_{\text {tube }} / g=1\right) / g^{2}=1$ and attain a plateau $g_{\text {tube }}\left(s_{\text {tube }}\right) / g^{2}=a / g$ by definition of tube Kuhn step $a$. Deviations at very large $s_{\text {tube }} \sim L$ are due to sampling bias and should be ignored. Indeed, since not all chains have tubes with $s_{\text {tube }}>L$, the $d_{\text {tube }}\left(s_{\text {tube }}\right)$ is only sampled from the more compact chains which wrap around the obstacles, thus having longer tube but smaller than average end-to-end distance. One can see that this bias is pushed to larger $s_{\text {tube }}$ as the chain length increases. The most interesting feature of these curves is their qualitatively different shape for $g=1$ as compared with the larger grids. Grid $g=1$ shows expected increase from $s / g=1$ to $s / g=2$ similar to what is expected from the non-returning random walk. After that, $d(s)$ reaches the plateau straight away. In contrast, $d(s)$ for larger grids has an extended decreasing region (up to $s / g \approx 20$ ). This is connected with the fact that tube axis has higher than expected probability to do a 90 degrees turn as compared to going straight. For a cubic grid with coordination number 6 we expect the probability going straight to be $1 / 5$. However, the measured probability is close to 0.1 for large grid sizes.

Fig. 7 shows the tube Kuhn steps $a_{t a}$ and $a_{p p}$ extracted from the average tube lengths using the tube axis and the primitive path constructions, respectively. The results can be extrapolated to infinite $N$ if plotted vs. $g^{2} / N$ and follows from the intersection of the linear fit with the vertical axis. If $a \approx g, b_{3 \mathrm{D}}^{2}=1$, then $g^{2}$ is the expected value of $N_{e}$ (eq. 15 ) and $g^{2} / N$ is the expected inverse number of entanglements, $\approx 1 / Z$. Table 1 and Fig. 8 provide the extrapolated values for different grid sizes. One can see that for large grids both definitions give the tube step proportional to the grid spacing $g$, however $a_{t a} \approx g / 2$ whereas $a_{p p} \approx 2 g$. Such a huge difference correspond to a factor of 16 difference in $N_{e}$, which means that one or both results do not make sense. Fig. 9 shows the plateau modulus extracted from $G(t)$ by fitting it with the slip-springs model, ${ }^{15}$ which provides a convenient extrapolation to $N \rightarrow \infty$. ${ }^{16}$ We compare it with the usual predictions from the equation

$$
G_{N}^{(0)}=\frac{4}{5} \frac{c k_{B} T}{a^{2} / b_{3 \mathrm{D}}^{2}}
$$

which follows from eq. 14 using $N_{e}=a^{2} / b_{3 \mathrm{D}}^{2}$ and $a$ extracted from the two definitions. It is clear that neither prediction is satisfactory - the only case where it might work with reasonable accuracy is for large grids $g \gg 1$ if the primitive path construction is used.

Another standard method to extract the tube Kuhn step $a$ is from the monomer MSD. In the conventional tube theory one assumes that $b_{1 \mathrm{D}}=b_{3 \mathrm{D}}$ and $\zeta_{1 \mathrm{D}}=\zeta_{3 \mathrm{D}}$, and therefore $a$ can be determined by two methods: from the transition around $\tau_{e}$ and from the transition around $\tau_{R}$. If one fits the middle monomer MSD with 
Table 1: The extrapolated values of $N_{e}$ for various grid sizes, obtained by different methods.

\begin{tabular}{|c|c|c|c|c|c|c|}
\hline \multirow{2}{*}{$\mathrm{g}$} & \multicolumn{7}{|c|}{$N_{e}$} \\
\cline { 2 - 7 } & TA & PP & $G_{n}^{0}$ & MSD around $\tau_{e}$ & MSD around $\tau_{R}$ & $S(q, t)$ \\
\hline 1 & 1.3 & 8.7 & 4.0 & 4.3 & 3.8 & 4.84 \\
2 & 2.7 & 23.7 & 12.7 & 10.7 & 14.5 & 14.8 \\
4 & 6.7 & 72.7 & 39 & 31.1 & 63.2 & 54.8 \\
8 & 18.2 & 248 & & 119 & & \\
16 & 58.1 & & & & & \\
32 & 187 & & & & & \\
64 & 614 & & & & & \\
\hline
\end{tabular}

the three predicted power laws in corresponding regimes

$$
g_{1, \text { mid }}(t) \approx\left\{\begin{array}{cc}
K_{0} t^{1 / 2}, & t \ll \tau_{e} \\
K_{1} t^{1 / 4}, & \tau_{e} \ll t \ll \tau_{R}^{1 \mathrm{D}} \\
K_{2} \frac{t^{1 / 2}}{\sqrt{N}} & t \gg \tau_{R}^{1 \mathrm{D}}
\end{array}\right.
$$

one can express the tube parameters from the second and the third regimes as

$$
\begin{aligned}
a b_{1 \mathrm{D}} & =\frac{\pi \sqrt{3}}{2} \frac{K_{1}^{2}}{K_{2}} \\
\tau_{0}^{1 \mathrm{D}} & =\frac{1}{\pi}\left(\frac{K_{1}}{K_{2}}\right)^{4}
\end{aligned}
$$

which for conventional tube theory simplifies to

$$
a=\frac{\pi \sqrt{3}}{2} \frac{K_{1}^{2}}{K_{2}}
$$

From the first and second regimes one can extract the tube step as

$$
a^{2} \frac{b_{1 \mathrm{D}}^{2}}{b_{3 \mathrm{D}}^{2}} \sqrt{\frac{\tau_{3 \mathrm{D}}}{\tau_{1 \mathrm{D}}}}=\frac{3 \pi}{2} \frac{K_{1}^{2}}{K_{0}}
$$

which simplifies for conventional tube theory to

$$
a=\sqrt{\frac{3 \pi}{2}} \frac{K_{1}}{\sqrt{K_{0}}}
$$

The predictions of $N_{e}=a^{2} / b_{3 \mathrm{D}}^{2}$ from eqs 19 and 20 are also plotted in Fig. 9. We see that neither of them 


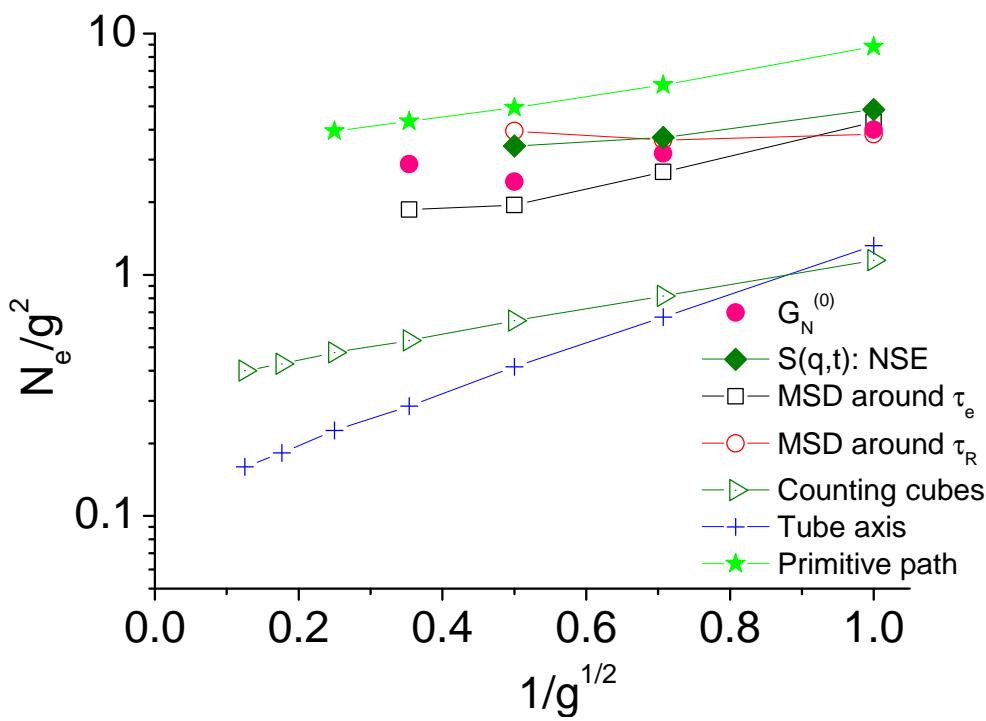

Figure 9: $N_{e}$ for the grid model obtained by a variety of traditional methods from the literature.

agrees with the plateau modulus, and results from eqs. 19 and 20 differ by a factor of 2 for $g=4$. The tube step extracted from the transition around $\tau_{R}$ (eq. 19) is larger than the one extracted from the transition around $\tau_{e}$ (eq. 20). This effect was observed in molecular dynamics simulations in ref. ${ }^{17}$ and was interpreted as an increase of tube diameter as time progresses in agreement with the dynamic tube dilution (DTD) picture. ${ }^{1}$ The dynamic dilution is caused by constraint release from the other chains, which is completely absent in our model. This means that the DTD interpretation might be ambiguous and an alternative explanation is needed, at least for the grid model.

For completeness we have added to Fig. 9 two more methods used in the literature as measures of $N_{e}$. One comes just from counting the tube segments. In other words, one sometimes assumes that $Z$ is just the number of tube segments (cubes making a tube axis in our case) instead of measuring the tube length and using eq. 8 to compute $Z$. We indicate $N_{e}$ obtained this way by triangles in Fig. 9 . One can see that this definition leads to results different from tube axis definition, which is clearly a consequence of non-ideal statistics of the tube axis (as illustrated in Fig. 6). Another measure of $N_{e}$ can be obtained by fitting the dynamic structure $S(q, t)$ with the empirical formula

$$
S(q, t) / S(q, 0)=\left[1-\exp \left(-\frac{g^{2} a^{2}}{36}\right)\right] S^{l o c}(q, t)+\exp \left(-\frac{q^{2} a^{2}}{36}\right) S^{\sec }(q, t)
$$

where $q$ is a scattering vector, $S^{l o c}(q, t)$ is the part of structure factor relaxation due to local monomer rearrangement inside the tube, and $S^{e s c}(q, t)$ is the part due to escape from the tube. An exact expression in the 


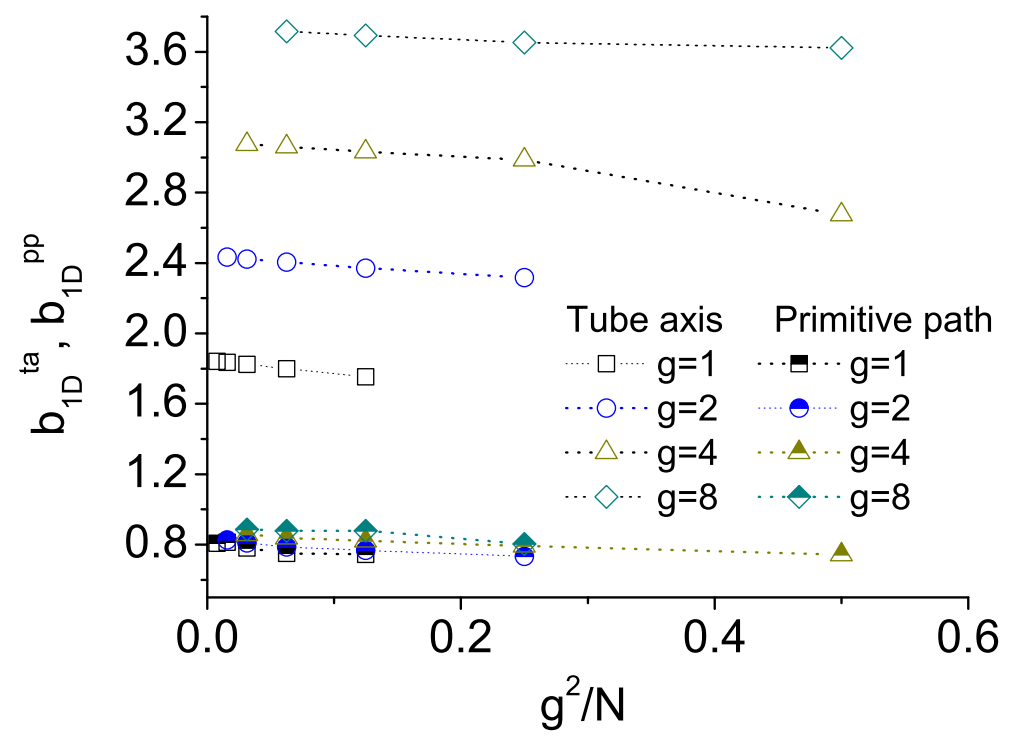

Figure 10: $b_{1 \mathrm{D}}$ as a function of $g^{2} / N$ from the fluctuations in the tube axis and primitive path length (eq. 22)

limit $q a \ll 1$ was derived by de Gennes, ${ }^{18}$ which had $\left(1-q^{2} a^{2} / 36\right)$ instead of exponentials. However the only experimental method to measure $S(q, t)$ is the neutron spin-echo (NSE), which is limited by the longest time of 200ns. This means that it is only possible to measure relaxation at $q a \sim 1$, because the smaller $q$ values do not show any appreciable signal. Thus eq. 21 is used instead, which should be treated as convenient fitting formula rather than an exact tube theory result. $N_{e}$ is then obtained as $a^{2} / b_{3 \mathrm{D}}^{2}$. We see that the value of $N_{e}$ obtained by determining $a$ from eq. 21 is about $40 \%$ larger than the one obtained from the plateau modulus. Similar results were reported for NSE measurements of polyethylene. ${ }^{19}$

Apart from the average tube length, leading to determination of $a$, one can extract more information from the variance of the length distribution $\left\langle\Delta L^{2}\right\rangle$. According to the Rouse theory, the variance of the end-to-end distance of the 1D Rouse chain stretched by the ends does not depend on the stretching force and is equal to

$$
\left\langle\Delta L^{2}\right\rangle=\frac{N b_{1 \mathrm{D}}^{2}}{3}
$$

where the factor of 3 is due to the fact that $L$ is measured in one dimension only. Thus, the measured variance provides a direct measure of $b_{1 \mathrm{D}}$ independently of $a$. The results can be again plotted as a function of $g^{2} / N$ and extrapolated to $N \rightarrow \infty$ for each grid size and for both methods (Fig. 10). The extrapolated values are plotted in Fig. 11, showing that the two methods give drastically different results. The extrapolation to infinite grid carries large error bars since they are already a result of another extrapolation $(N \rightarrow \infty)$, but it looks like for large grids the difference can be around a factor of 7. This means that the amount of contour 


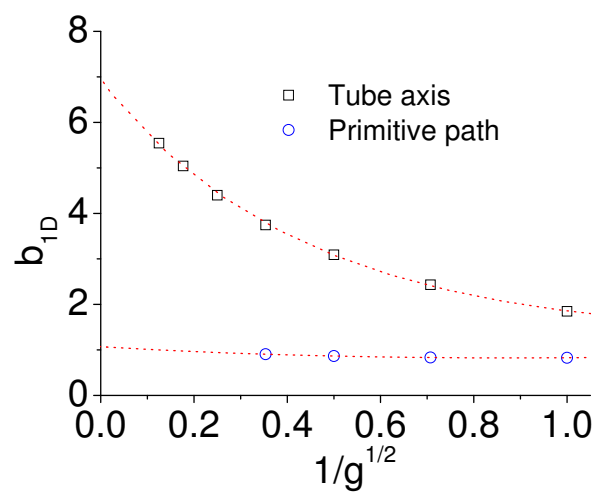

Figure 11: Extrapolated $b_{1 \mathrm{D}}$ from the tube axis and primitive path.

length fluctuations (CLF) predicted by the two tube constructions is very different, which should reflect in stress relaxation and all other dynamic quantities.

A solution to this problem is to consider instead the relative amount of tube length fluctuations

$$
\frac{\Delta L}{L}=\frac{a b_{1 \mathrm{D}}}{b_{3 \mathrm{D}}^{2}(3 N)^{1 / 2}}
$$

which follows from eqs. 7 and 22. This does not depend on the absolute value of $L$, and therefore the two differences might cancel out. It only depends on the combination $a b_{1 \mathrm{D}}$ and on the static properties of the chain $\left(N\right.$ and $\left.b_{3 \mathrm{D}}\right)$. Moreover, we saw from eq. 13 that the monomer MSD is also only affected by the combination $a b_{1 \mathrm{D}}$. Plotting this combination in Fig. 14, we find that the both tube axis and the primitive path methods provide almost identical results (if extrapolated values are used). This is very good news: despite the large uncertainty for each of the parameters individually, their product can be reliably extracted from either method. Thus, to predict the monomer MSD it does not matter which method of tube construction one uses. However, we need one more parameter $\tau_{0}^{1 \mathrm{D}}$ to give a definite prediction, unless one assumes that it is equal to $\tau_{0}^{3 \mathrm{D}}$. Next section examines this assumption and tests the static analysis predictions.

\section{Dynamic analysis}

We shall examine two different methods of extracting the tube parameters from the dynamic observables, namely measuring $g_{s}(t)$ (eqs. 4,6 ) or directly from $g_{1, \text { mid }}(t)$, eq. 13. For the first method (more elaborate and only possible for some models), we construct the tube axis at every time step during the simulation, project all beads onto this path, align the tube axis onto the tube axis from the past, and calculate how many tube segments did the middle monomer travel along the tube. The obtained results are plotted in Fig. 12 for 


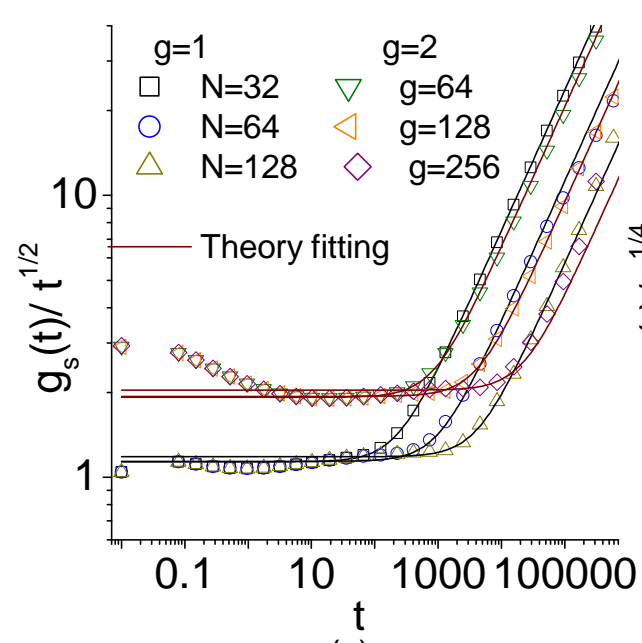

(a)

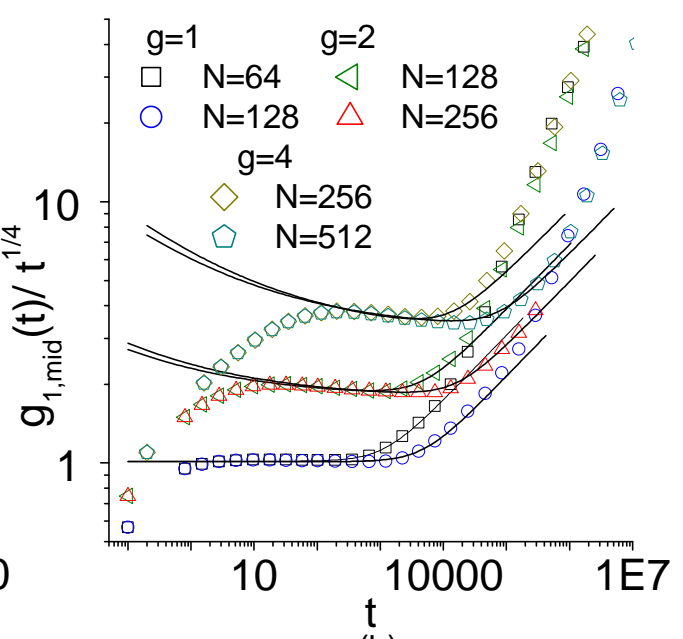

(b)

Figure 12: (a) $g_{s}(t)$ and the fitting line obtained from eq. 6 for various grid sizes and chain lengths. (b) $g_{1, \text { mid }}(t)$ and the fitting line obtained from eq. 24 for various grid sizes and chain lengths.

different grids and can be fitted with eq. 6 (lines). Notice however that the fit for times before the transition is not very convincing especially for $g=2$ due to the discreteness of the tube segment counting (we only resolved $\Delta s=n g$ with integer $n$ ). We found this method very time consuming, and giving the same results as the computationally cheaper method described below.

Another method is just fitting the $g_{1 \text {,mid }}(t)$ from Fig. 12 (b) around the transition from $t^{1 / 4}$ to $t^{1 / 2}$ regimes with eq. 13, and extract $a b_{1 \mathrm{D}}$ and $\tau_{0}^{1 \mathrm{D}}$ from the fit. In Fig. 12(b) $g_{1, \text { mid }}(t) / t^{1 / 4}$ is plotted and one can see that for large grid sizes the data show a small negative slope, meaning that the $g_{1 \text {,mid }}(t)$ grows slower than the $t^{1 / 4}$ power law. This can be easily explained by the motion perpendicular to the tube contour. We can reasonably assume that MSD can be approximately divided into a sum of displacements parallel and perpendicular to the tube contour. At times $t<\tau_{e}$ they will both grow proportional to $t^{1 / 2}$. After $\tau_{e}$, perpendicular displacement will saturate at some level of order $a^{2}$, whereas the parallel displacement will grow as $t^{1 / 4}$. Therefore the appropriate fitting formula in the regime between $\tau_{e}$ and $\tau_{R}^{1 \mathrm{D}}=N^{2} \tau_{0}^{1 \mathrm{D}}$ is $c+K_{1} t^{1 / 4}$, which indeed has a small negative slope if divided by $t^{1 / 4}$. Using the approximate equation for $g_{s}(t)$, eq. 6 , we arrive at the following fitting formula

$$
g_{1, \text { mid }}(t) \approx c+a b_{1 \mathrm{D}} \sqrt{\frac{4}{3 \pi^{3}}}\left(\frac{t}{\tau_{0}^{1 \mathrm{D}}}\right)^{1 / 4}\left(\left(\frac{t}{N^{2} \tau_{0}^{1 \mathrm{D}}}\right)^{3 / 2}+\pi^{3 / 2}\right)^{1 / 6}
$$

The fitting parameter $c$ should be normalized by $g^{2}-$ the characteristic squared size of perpendicular 
fluctuations. Furthermore $a b_{1 \mathrm{D}}$ can be normalized by $g$ since the tube theory expects $a \sim g$ and $b_{1 \mathrm{D}} \approx$ const, $\tau_{0}^{1 \mathrm{D}}$ can be measured in units of $\tau_{0}^{3 \mathrm{D}}=\frac{1}{3 \pi^{2}}$ in simulation units. Hence $\tau_{0}^{1 \mathrm{D}} / \tau_{0}^{3 \mathrm{D}}=\frac{b_{1 \mathrm{D}}^{2} \zeta_{1 \mathrm{D}}}{b_{3 \mathrm{D}}^{2} \zeta_{3 \mathrm{D}}}$, and therefore

$$
\zeta_{1 \mathrm{D}}=\zeta_{3 \mathrm{D}} \frac{b_{3 \mathrm{D}}^{2} \tau_{0}^{1 \mathrm{D}}}{b_{1 \mathrm{D}}^{2} \tau_{0}^{3 \mathrm{D}}}
$$

The fits to eq. 24 are shown in Fig. 12(b), and the normalized fitting parameters are listed in Table 2 and plotted in Fig. 13. We also include the value for the 1D friction coefficients for the two tube methods using eq. 25. We found that for a more consistent and stable fitting we can fix $c$ for each grid independent of $N$, and found $c \approx 0$ for $g=1, c \approx 0.5$ for $g=2$ and $c \approx 3.0$ for $g=4$. Fig. 14 shows that the values of $a b_{1 D}$ extrapolated to $N \rightarrow \infty$ are in good agreement with static analysis result (within $20 \%$ as compared to factors 4-7 differences in $a$ and $b_{1 \mathrm{D}}$ separately). This provides a consistently check and confirms the universality of the product $a b_{1 \mathrm{D}}$. Note however that there are two remaining discrepancies. The $a b_{1 \mathrm{D}}$ extracted from the dynamical analysis is about $10 \%$ lower than the one obtained from the primitive path. In contrast, the result from the tube axis deviates from the monotonic behaviour and increases with increasing grid size, potentially deviating from two other methods significantly.

Table 2: Extrapolated values of the tube parameters from different methods.

\begin{tabular}{|c|c|c|c|c|c|c|c|c|c|c|}
\hline \multirow{3}{*}{ grid } & \multicolumn{2}{|c|}{ static analysis } & \multicolumn{8}{|c|}{$g_{1, \text { mid }}(t)$ fit } \\
\hline & \multirow{2}{*}{$\begin{array}{c}\text { tube axis } \\
a b_{1 \mathrm{D}}\end{array}$} & \multirow{2}{*}{$\begin{array}{c}\text { primitive path } \\
a b_{1 \mathrm{D}}\end{array}$} & \multirow{2}{*}{$a b_{1 \mathrm{D}}$} & \multirow{2}{*}{$b_{1 \mathrm{D}}^{2} \zeta_{1 \mathrm{D}}$} & \multicolumn{3}{|c|}{ tube axis } & \multicolumn{3}{|c|}{ primitive path } \\
\hline & & & & & $a$ & $b_{1 \mathrm{D}}$ & $\zeta_{1 D}$ & $a$ & $b_{1 \mathrm{D}}$ & $\zeta_{1 D}$ \\
\hline 1 & 2.1 & 2.48 & 2.05 & 2.88 & 1.15 & 1.85 & 0.85 & 3.0 & 0.83 & 4.35 \\
\hline 2 & 4.0 & 4.0 & 3.5 & 3.39 & 1.65 & 2.44 & 0.45 & 4.8 & 0.83 & 3.96 \\
\hline 4 & 8.0 & 7.5 & 7.02 & 4.13 & 2.59 & 3.09 & 0.25 & 8.7 & 0.86 & 3.23 \\
\hline 8 & 16.3 & 14.8 & & & & & & & & \\
\hline 16 & 34 & & & & & & & & & \\
\hline 32 & 70 & & & & & & & & & \\
\hline 64 & 142 & & & & & & & & & \\
\hline
\end{tabular}

Fig. 15(a) shows the effective Rouse time in one dimension, $\tau_{R}^{1 \mathrm{D}}$ which is one of the key results of this paper. We see that the Rouse time inside the tube is larger than the free Rouse time by a factor of 3-4. This explains the discrepancy between the tube steps obtained from fitting $g_{1 \text {,mid }}(t)$ around $\tau_{e}$ and around $\tau_{R}$ (Fig. 9), which was a consequence of the assumption $\tau_{0}^{1 \mathrm{D}}=\tau_{0}^{3 \mathrm{D}}$.

This result is independent of the tube construction, but its interpretation of course will be different in different tubes. If $b_{1 \mathrm{D}}$ is obtained from the static tube axis analysis, it will result in $\zeta_{1 \mathrm{D}}<\zeta_{3 \mathrm{D}}$ (plotted in Fig. 15(b)). This means that the motion along the tube axis is faster than expected. Since the motion in the environment with obstacles can only be slower than the free motion, one has to conclude that the tube axis 


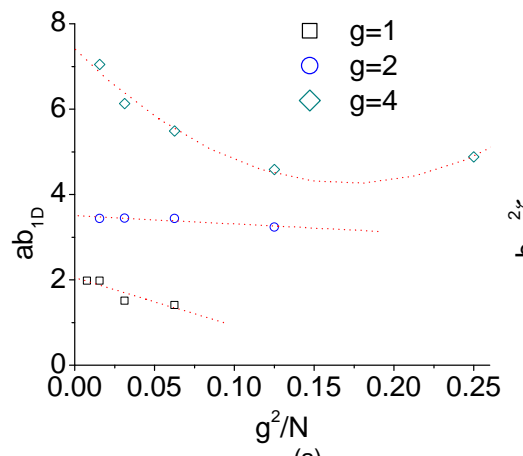

(a)

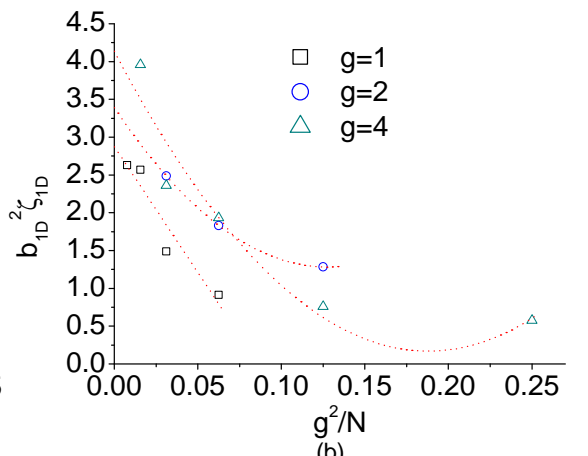

(b)

Figure 13: (a) $a b_{1 \mathrm{D}}$ for various grid sizes and (b) $b_{1 \mathrm{D}}^{2} \zeta$ for various chain length and grid sizes. Dotted lines are a guide to the eye.

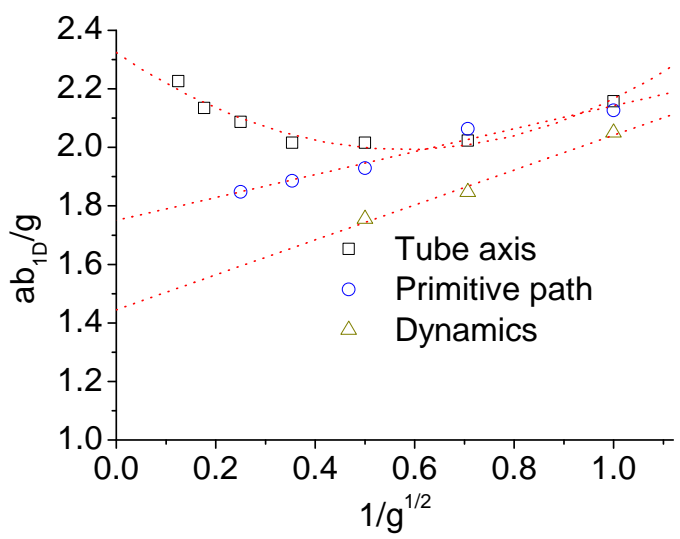

Figure 14: The value of $a b_{1 \mathrm{D}}$ for $N \rightarrow \infty$ as a function of $g^{-1 / 2}$. Dotted lines are a guide to the eye. 

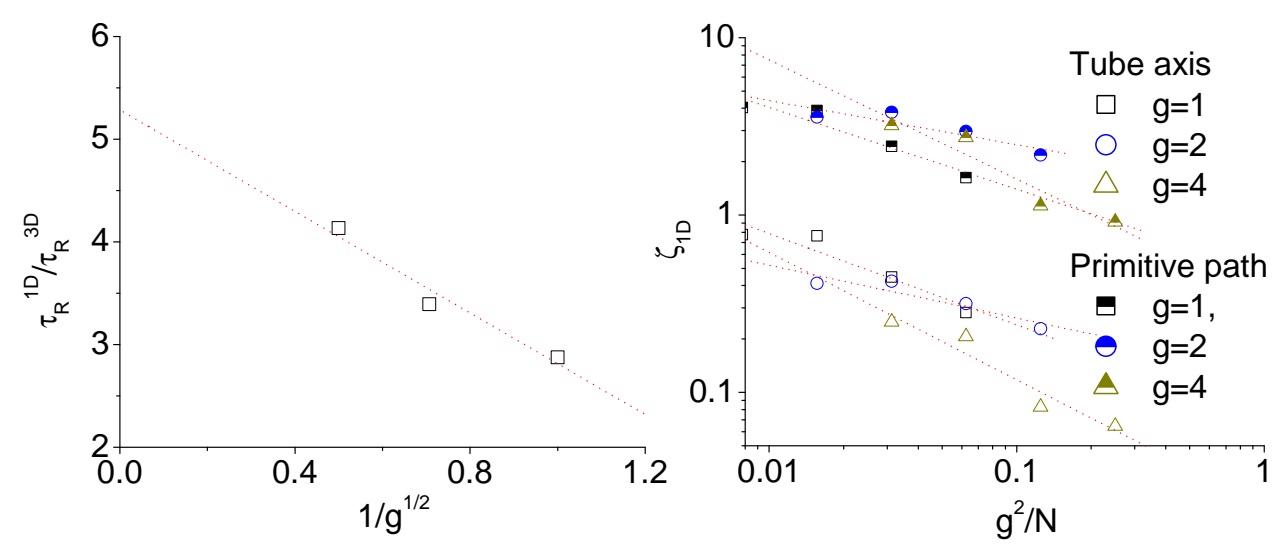

Figure 15: (a)Asymptotic values of the Rouse time inside the tube $\tau_{\mathrm{R}}^{1 \mathrm{D}}$ normalised by its free counterpart $\tau_{R}^{3 \mathrm{D}}$, (b) $\zeta_{1 \mathrm{D}}$ based on the tube axis and the primitive path.

method overestimates the actual distance the chain has to diffuse to escape from the tube. In contrast, if $b_{1 \mathrm{D}}$ is extracted from the primitive path analysis, the effective $\zeta_{1 \mathrm{D}}$ is significantly larger than $\zeta_{3 \mathrm{D}}$. The limiting value for $N \rightarrow \infty$ is about 4 for $g=1$ and 2. This implies that either the diffusion along the primitive path is slowed down by the obstacles, that the actual diffusion path is longer than the primitive path, or most likely a combination of both.

\section{Discussion}

In the previous sections we showed that the tube model in general has at least three parameters: the tube Kuhn step $a$, the one-dimensional statistical segment $b_{1 \mathrm{D}}$ and the effective one-dimensional friction inside the tube $\zeta_{1 \mathrm{D}}$. However, the monomer diffusion inside the tube is controlled by two combinations of these parameters $a b_{1 \mathrm{D}}$ and $\tau_{0}^{1 \mathrm{D}}=\frac{b_{1 \mathrm{D}}^{2} \zeta_{1 \mathrm{D}}}{3 \pi^{2} k_{B} T} \sim b_{1 \mathrm{D}}^{2} \zeta_{1 \mathrm{D}}$. Although $a$ and $b_{1 \mathrm{D}}$ obtained by different tube constructions are very different, their product is almost the same and agrees well with $a b_{1 \mathrm{D}}$ obtained by direct fitting of $g_{1, \text { mid }}(t)$. We believe this statement remains valid even at timescales when the chain escapes from the tube. Indeed, let's consider predictions of the tube theory with three parameters for reptation time $\tau_{d}^{(0)}(N)$ without CLF. Since the result will not depend on $b_{1 \mathrm{D}}$, one might wonder if the described invariance is broken. According to the solution of the simple 1D diffusion equation, ${ }^{9}$ the escape time from the domain of length $L$ 
with diffusion coefficient $D_{c}$ is

$$
\begin{aligned}
\tau_{d}^{(0)} & =\frac{L^{2}}{\pi^{2} D_{c}}=3 \tau_{R}^{1 \mathrm{D}} \frac{N}{\tilde{N}_{e}} \\
\tilde{N}_{e} & \equiv \frac{a^{2} b_{1 \mathrm{D}}^{2}}{b_{3 \mathrm{D}}^{4}}
\end{aligned}
$$

where we used the definition of $a$, eq. 7 and $D_{c}=\frac{k_{B} T}{N \zeta_{1}}$ is the center of mass diffusion coefficient inside the tube. In this form the equation looks similar to the standard tube theory formula $\tau_{d}^{(0)}=3 \tau_{R} N / N_{e}$, however the Rouse time must be one-dimensional, and $N_{e}$ must be replaced by a combination of $a$ and $b_{1 \mathrm{D}}$ (instead of the usual $N_{e}=a^{2} / b_{3 \mathrm{D}}^{2}$ ). To find corrections to this expression due to CLF, we replace (following Doi ${ }^{20}$ ) $L$ by $L-C^{\prime} \Delta L$ in eq. 26 where $\Delta L=\sqrt{\left\langle\Delta L^{2}\right\rangle}=b_{1 \mathrm{D}} \sqrt{N / 3}$. This leads to

$$
\tau_{d} \approx 3 \tau_{R}^{1 \mathrm{D}} \frac{N}{\tilde{N}_{e}}\left(1-C^{\prime} \frac{\Delta L}{L}\right)^{2}=3 \tau_{R}^{1 \mathrm{D}} \frac{N}{\tilde{N}_{e}}\left(1-C \sqrt{\frac{\tilde{N}_{e}}{N}}\right)^{2}
$$

where $C^{\prime}=\sqrt{3} C$ (using eq. 23) and $C$ is a numerical constants of order unity (numerical simulations show that $C \approx 1.7^{12}$ ). Thus, the same combination $\tilde{N}_{e}$ appears in both the leading term and the first order correction term as in the $g_{1, \text { mid }}(t)$ expression. Note however that the second order corrections to eq. 28 will depend on the tube properties on the length scale of the tube step $a$, and will be characterized by other parameters. We see that the combination $\tilde{N}_{e}$ plays an important role in the tube theory. We shall call it the invariant number of segments between entanglements, or the invariant $N_{e}$.

However not all dynamic observables can be predicted from $\tilde{N}_{e}$ and $\tau_{0}^{1 \mathrm{D}}$ — one notable exception is the plateau modulus, the amplitude of the stress relaxation function $G(t)$ in the limit of long chains. Let's recall the tube theory expression for the stress tensor. ${ }^{9,11}$ It assumes a picture of slip-links or tube vertices $\left\{\mathbf{V}_{i}\right\}$ fixed in space, with the chain having to thread through these slip-links and having $n_{i}$ monomers between slip-links $\mathbf{V}_{i}$ and $\mathbf{V}_{i+1}$. This leads to the stress expression

$$
\sigma_{\alpha \beta}=-\frac{1}{V} \sum_{\text {interacting pairs } p} F_{\alpha}(p) r_{\beta}(p)=-\frac{1}{V} \sum_{j=1}^{N_{c}} \sum_{i=0}^{L / a-1} \frac{3 k_{B} T}{b_{1 \mathrm{D}}^{2}} \frac{\left(\mathbf{V}_{i+1}-\mathbf{V}_{i}\right)_{\alpha}\left(\mathbf{V}_{i+1}-\mathbf{V}_{i}\right)_{\beta}}{n_{i}}
$$

Here we used microscopic stress definition as a sum over all pairs $p$ of particles with non-zero pairwise force $\mathbf{F}(p)$ and connector vector $\mathbf{r}(p)$. To calculate the force between points $\mathbf{V}_{i+1}$ and $\mathbf{V}_{i}$ we used the spring constant $3 k_{B} T /\left(n_{i} b_{1 \mathrm{D}}^{2}\right)$, since $b_{1 \mathrm{D}}$ is the relevant statistical segment inside the tube. $V$ is the system volume and $N_{c}$ is the number of chains in this volume. They are connected with the monomer concentration $c$ by 


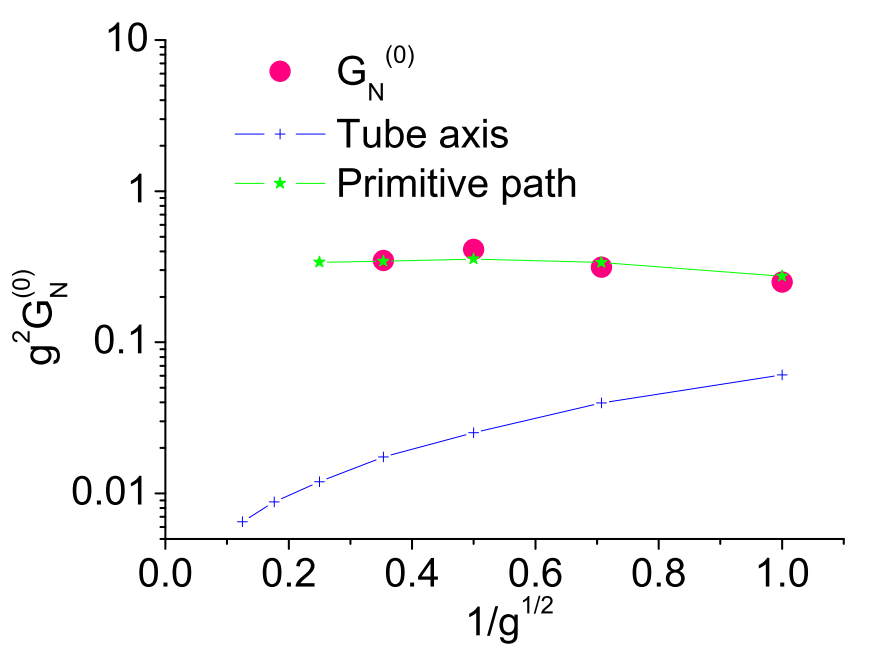

Figure 16: $g^{2} G_{N}^{(0)}$ from the tube axis, primitive path and direct fit of $G(t)$ by the slip-springs model

$c=N N_{c} / V$. To estimate the amplitude of the plateau modulus $G_{N}^{(0)} \approx G\left(\tau_{e} \ll t \ll \tau_{d}\right)$ (without the numerical coefficient), we use the fluctuation-dissipation theorem

$$
G(t)=\frac{V}{k_{B} T}\left\langle\sigma_{x y}(t) \sigma_{x y}(0)\right\rangle \sim \frac{k_{B} T N_{c}}{V b_{1 \mathrm{D}}^{4}} \frac{L}{a} a^{4}\left\langle\frac{1}{n_{i}(t)} \frac{1}{n_{i}(0)}\right\rangle
$$

Here we have assumed that the different tube segments are uncorrelated with each other and all have length $a$, and are not renewed on the time scale $t \ll \tau_{d}$. Now we can assume that $n_{i}\left(t \gg \tau_{e}\right)$ is uncorrelated with $n_{i}(0)$. Replacing $\left\langle\frac{1}{n_{i}(t)} \frac{1}{n_{i}(0)}\right\rangle$ by $1 /\left\langle n_{i}\right\rangle^{2}=b_{3 \mathrm{D}}^{4} / a^{4}$ (eq. 15), we get

$$
G_{N}^{(0)} \sim c k_{B} T \frac{b_{3 \mathrm{D}}^{6}}{b_{1 \mathrm{D}}^{4} a^{2}}=\frac{c k_{B} T}{\tilde{N}_{e}} \frac{b_{3 \mathrm{D}}^{2}}{b_{1 \mathrm{D}}^{2}}
$$

This shows that according to the tube theory the plateau modulus depends on a different combination of $a$ and $b_{1 \mathrm{D}}$ as compared to all other quantities. Therefore although the shape of $G(t)$ at $t \gg \tau_{e}$ will probably be controlled by $\tilde{N}_{e}$ only, its amplitude has an additional factor $\frac{b_{3 \mathrm{D}}^{2}}{b_{1 \mathrm{D}}^{2}}$. This would mean that the tube theory prediction for the plateau modulus will depend on the tube construction, and two different constructions considered above will give very different predictions. These predictions are shown in Fig. 16 by lines with small symbols, ignoring the fact we do not know the prefactor in eq. 31 and setting it to 1 . Filled circles show $G_{N}^{(0)}$ obtained by fitting $G(t)$ of the grid model with $G(t)$ of the slip-springs model (as described before). The tube axis predictions are terrible, underestimating plateau modulus by a factor of up to 100 for large grids. Although the prediction from the primitive path construction shows very good agreement 


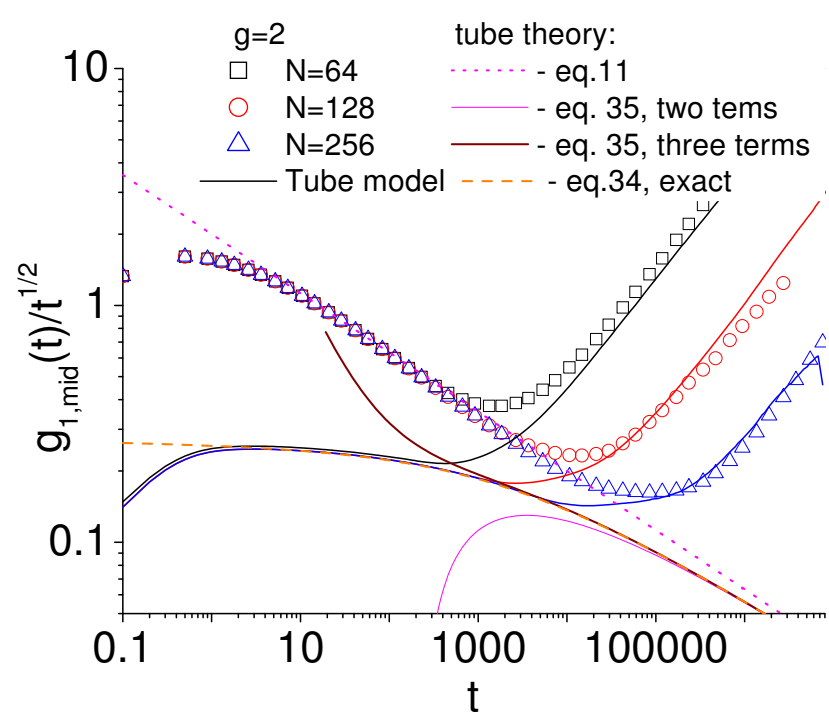

(a)

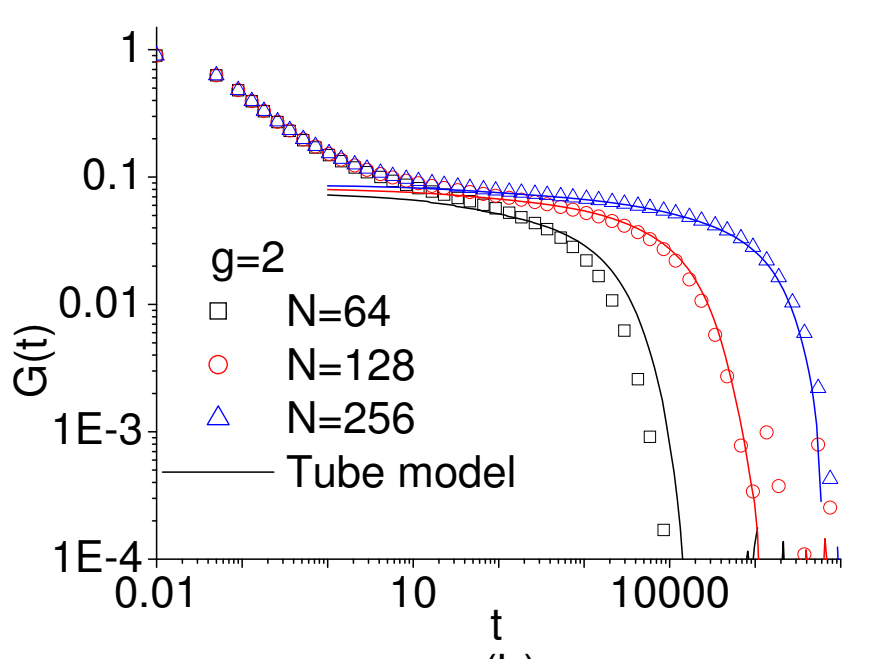

(b)

Figure 17: Comparison of the grid model (symbols) for $g=2$ with $N=64,128,256,512$ and the tube model (solid lines) where the input parameters are $a=4.812, b_{1 \mathrm{D}}=0.833, \zeta_{1 D}=3.964$.

with $G_{N}^{(0)}$ measurement, we have to be aware that it can be a simple coincidence. Indeed, let's compare the assumptions behind this prediction with the assumptions used in the derivation of the monomer MSD. In obtaining eq. 13 we only used the fact that the tube has random walk statistics at long scales, much larger than $a$, and thus if the monomer has moved the distance $\Delta s$ along the tube, its real space square displacement is $a \Delta s$. No assumptions on the length scale of the tube were made, and in fact the fits on these length scale were not satisfactory. Fig. 6 showed significant deviations from the random walk statistics for small $\Delta s$.

In contrast, eq. 29 contains many assumptions exactly on the scale of one tube segment, and these assumptions are quite unphysical. Indeed, it is strange to assume that the chain has to pass exactly through an infinitely small point in space at all moments of time, and that all tube segments have uncorrelated directions and equal lengths. Numerically it is easy to show that changing these postulates results in significant changes of the plateau modulus. Besides, the prefactor in eq. 31 is affected by the longitudinal redistribution of monomers along the tube and the correlation function of $1 / n_{i}(t)$ must be carefully evaluated and will depend on the model used. Therefore in our opinion, the described tube theory is adequate to predict the monomer diffusion and the shape of other relaxation functions for $t \gg \tau_{e}$, but is not microscopic enough to predict the stress plateau modulus unambiguously. We note however that although the prefactor in eq. 31 is wrong, it seems that the primitive path is closer to the "real tube" than the tube axis, as shown in Figs. 11, 14 and 16.

Finally, we perform tube model simulations with parameters $a, b_{1 D}$ and $\xi_{1 D}$ extracted from the primitive path simulations. The tube model simulation consists of a one-dimensional stretched Rouse chain simulation, 
which is projected every step onto a three dimensional freely-jointed random walk, which represents the tube. Tube segments are deleted and inserted at the ends depending on the chain end position (see ref. ${ }^{11}$ for detailed model description). The results of such tube model simulation are compared with the grid model results $(g=2)$ in Fig. 17. We see that although the agreement of stress relaxation is very good, the agreement for the MSD is surprisingly poor. The two models start to agree only after $t \approx 10^{4}$. This is about 500 times larger than $\tau_{e} \approx 20$ defined as $a^{4} \tau_{0}^{3 D}$. In fact, the tube model simulation hardly shows any sign of $t^{1 / 4}$ behaviour for the chain lengths simulated (up to about 10 entanglements). However our mapping procedure guarantees that these two models will agree perfectly in the limit of very long chains. This indicates that for the MSD the convergence to this limit is extremely slow. In fact it is quite a surprise that the grid model agrees with the tube theory predictions, eq. 13, much better than the tube model (dotted line in Fig.17(a)). To investigate the reasons for the disagreement between the tube model and the tube theory, we perform detailed analytical calculations of monomer MSD for the freely-jointed tube model, Appendix B. We show that this is due to next order correction terms in eq. 9. Indeed, replacing it with the exact expression leads to the perfect agreement with the tube model simulations (dashed line in Fig.17(a)).

Even more surprising is that the grid model shows almost perfect $t^{1 / 4}$ behaviour. We believe this is due to the effect of motion perpendicular to the tube contour, which partly cancels the negative corrections derived

in Appendix B. This means that the apparent $t^{1 / 4}$ regime shown by the grid model (as well as by many other models including the slip-springs model and MD simulations of the Kremer-Grest bead-spring model) is actually coincidental. Indeed, for larger grid spacing and for slip-springs model we see more pronounced deviations from the $t^{1 / 4}$ scaling. The tube model will show an ideal behaviour only for much longer chains, but the addition of perpendicular fluctuations makes it look like it is already present for shorter chains of less than 10 entanglements.

\section{Conclusions}

We have reported extensive simulations of a very simple model for an entangled chain: a Rouse chain in a cubic array of line obstacles. Despite its simplicity, the model has several advantages. At small times the model exhibits perfect Rouse behavior with the known friction $\zeta_{3 \mathrm{D}}$ and statistical segment $b_{3 \mathrm{D}}$. At longer times it shows the stress relaxation plateau as well as $t^{1 / 4}$ and $t^{1 / 2}$ regimes of the monomer MSD. The absence of constraint release (CR) is an additional advantage from the tube theory modelling point of view. Indeed, the comparison is not obscured by the difficult-to-control CR effect. Thus, our grid model provides a simple 
test ground for modern algorithms of the tube parameter estimation. One expects a perfect correspondence between this model and the tube theory, with only one parameter $a$ left to determine for each grid size $g$. In fact, it is reasonable to anticipate that $a \approx g$.

Contrary to these expectations, we show that despite the usual believe that the tube theory contains only one parameter describing entanglements (namely $a$ or $N_{e}$ ), one might have up to three such parameters: tube Kuhn step $a$, monomer friction inside the tube $\zeta_{1 \mathrm{D}}$ and statistical segment inside the tube $b_{1 \mathrm{D}}$. For each definition of the tube these parameters will be different. Indeed, we tried two such definitions (the tube axis and the primitive path) and found that all three parameters are vastly different (factors of 2-5 or even 10 in the limit of large grids). It is not clear to us whether there is a single correct definition of the tube length. However we noticed that the mean-square monomer displacement at long times $t \gg \tau_{e}$ depends only on two combinations of these three parameters, namely on the Rouse time inside the tube $\tau_{R}^{1 \mathrm{D}}$ and on the invariant number of entanglements $\sqrt{\tilde{N}_{e}}=\frac{a b_{1 \mathrm{D}}}{b_{3 \mathrm{D}}^{2}}$. Moreover, the relative fluctuations in the tube length is only controlled by this parameter. Interestingly, estimates for $\tilde{N}_{e}$ come out almost the same (within $10 \%$ ) from three different methods: two tube construction methods and from the direct fitting of the monomer MSD. This is encouraging news: it may be that the invariant number of entanglements is much less sensitive to the details of the tube construction as compared to the usual $N_{e}$ determined from the average tube length only. Thus, our suggestion to the primitive path analysis is to compute the first two moments of the tube length distribution $\langle L\rangle$ and $\left\langle L^{2}\right\rangle$, instead of just $\langle L\rangle$ as is routinely done, and therefore determine both $a$ and $b_{1 \mathrm{D}}$. If it happens that $b_{1 \mathrm{D}}=b_{3 \mathrm{D}}$, then the old results obtained from the average length are valid. If it is not the case, the shapes of all relaxation curves will be affected.

Another major conclusion of our study is that the Rouse time inside the tube $\tau_{R}^{1 \mathrm{D}}$ might not be equal to the one of the free chain without entanglements $\tau_{R}^{3 \mathrm{D}}$. For our model it is larger by about a factor of 3 in the long-chain limit. Such large discrepancy strongly affects the shapes of all relaxation curves and invalidates earlier $N_{e}$ estimates obtained from the monomer MSD or similar methods. The major challenge now is to measure $\tau_{R}^{1 \mathrm{D}}$ for more realistic models. Unfortunately, one requires relatively long chains to identify the $t^{1 / 4}$ and $t^{1 / 2}$ regimes clearly. The correction due to CR must be also taken into account. We leave these studies for future publications.

We note here briefly the connection of our parameter $b_{1 \mathrm{D}}$ with the CLF potential parameter $v$ used in the literature. The efective potential controlling CLF, proposed by Doi and Kuzuu, ${ }^{21}$ has the form

$$
U(L)=v Z\left(1-\frac{L}{\langle L\rangle}\right)^{2}
$$


Comparing it with the Gaussian distribution $P(L) \sim \exp \left(-U(L) / k_{B} T\right)$, we find that in our notations

$$
v=\frac{3}{2} \frac{b_{3 \mathrm{D}}^{2}}{b_{1 \mathrm{D}}^{2}}
$$

If, for example, $b_{1 \mathrm{D}} \approx 3 b_{3 \mathrm{D}}$ (as for the tube axis construction for $g=4$ ), then this implies that $v \approx \frac{3}{2} \times \frac{1}{9}$, i.e., much smaller than the expected $\frac{3}{2}$. This result, however, does not make much sense. Indeed, we showed that only the combination $\sqrt{\tilde{N}_{e}}=a b_{1 \mathrm{D}} / b_{3 \mathrm{D}}^{2}$ has a physical significance, which translates into

$$
\tilde{N}_{e}=\frac{a^{2}}{b_{3 \mathrm{D}}^{2}} \frac{3}{2 v}
$$

This means that if the measured $v$ is different from $3 / 2$, the invariant number of entanglements $\tilde{N}_{e}$ must be corrected to take this into account. The connection of $v$ and $b_{1 \mathrm{D}}$ for slip-link model is discussed in more detail in Appendix A.

A separate note is due about the stress plateau calculations. The primitive path analysis was invented to predict the plateau modulus using eq. 17. This equation is based on numerous assumptions which are quite unphysical. We therefore think that this is not adequate and that the tube theory does not contain enough details to predict the stress amplitude quantitatively. This is to be contrasted with the shape of the stress relaxation curve for $t \gg \tau_{e}$, where the tube theory might do a good job. The main reason is that to derive an equation of the sort of eq. 17 one requires explicit assumptions about the tube configuration and the tube potential on the length scale of the tube Kuhn step $a$. It is clear that such information is non-trivial: in all tube construction algorithms the resulting tube statistics deviates from the ideal random walk on these lengthscales. More explicitly, the tube steps are variable and the step directions are correlated. Following a simple calculation outlined in the previous section, it is easy to see that these deviations will affect the prefactors in eq. 31. Moreover, even the scaling $G_{N}^{(0)} \sim 1 / N_{e}$ or $G_{N}^{(0)} \sim 1 / \tilde{N}_{e}$ is not confirmed, and the plateau modulus might depend on a different combination of $a$ and $b_{1 \mathrm{D}}$. Thus, we think that the current tube theory can not be used for correctly predicting the plateau modulus in a quantitative way. In contrast, it seems that the slip-spring model ${ }^{22}$ does a very good job in predicting the stress amplitude of molecular dynamics data of monodisperse melts, once the monomer MSD is fitted. 


\section{Appendix A: Slip-link model of Schieber et al.}

Here we apply our definitions to the slip-link model ${ }^{23}$ and address some of its unexplained results. The slip-link model of Schieber et. al., ${ }^{2324}$ assumes the following tube statistics: one first generates a Gaussian polymer chain, then selects the number of slip-links $Z_{S}$ from the Poisson distribution with the single parameter $\beta$ determining the average number of slip-links per chain, and then selects $Z_{S}$ monomers from the uniform distribution $[0 . . N]$, where $N$ is the number of statistical segments in the polymer chain. (We note in passing that the notion of a Kuhn segment used in ref. ${ }^{24}$ does not exist for the Gaussian chain since such chain does not have a maximum contour length. The statistical segment must be used instead.) The positions of these $Z_{S}$ monomers then determine the positions of the tube vertices $\left(\mathbf{V}_{i}\right.$ in our notations). The tube length is then uniquely determined by this construction as

$$
L=\sum_{i=1}^{Z_{s}-1}\left|\mathbf{V}_{i+1}-\mathbf{V}_{i}\right|
$$

The first two moments of the tube length distribution for this model were calculated analytically in ref. ${ }^{24}$ In the limit of well entangled chains $N / \beta \gg 1$ they take a very simple form

$$
\langle L\rangle=\sqrt{\frac{2}{3}} \frac{N b_{3 \mathrm{D}}}{\sqrt{\beta}} ; \quad\left\langle\Delta L^{2}\right\rangle=\frac{N b_{3 \mathrm{D}}^{2}}{3}
$$

where ref. ${ }^{24}$ notations $N_{k}$ and $a_{K}$ are replaced by our notations $N$ and $b_{3 \mathrm{D}}$ respectively. Combining this with our definitions of $a$ and $b_{1 \mathrm{D}}$, we get

$$
a \equiv \frac{N b_{3 \mathrm{D}}^{2}}{\langle L\rangle}=\sqrt{\frac{3}{2} \beta} b_{3 \mathrm{D}} ; \quad b_{1 \mathrm{D}}^{2} \equiv \frac{3\left\langle\Delta L^{2}\right\rangle}{N}=b_{3 \mathrm{D}}^{2}
$$

The second equation suggests that for the model of Schieber et. al. the statistical segment inside the tube is equal to the statistical segment of the chain, i.e., the model shares the same assumption as the tube model. This seem to contradict the main conclusion of ref. ${ }^{24}$ that the parameter $v$ of the effective potential governing CLF is equal to 1 for large $Z$ rather than to $3 / 2$ as expected by the tube theory (see eq. 33 ).

The confusion is easily resolved by noticing that in the tube theory, once $L$ is defined, the so-called number of entanglements $Z$ must be defined through the average $L$ using eq. 8 . Substituting eq. 34 into eq. 8 leads to

$$
Z=\frac{2}{3} \frac{N}{\beta}
$$


This should be contrasted with the relation of $\beta$ and average $Z_{s}$, which is obtained from the Poisson probability distribution of $Z_{s}$ given in ref. ${ }^{23}$ In the limit of large $N / \beta$ it simplifies to

$$
\left\langle Z_{s}\right\rangle=\frac{N}{\beta}
$$

and thus

$$
Z=\frac{2}{3}\left\langle Z_{s}\right\rangle
$$

Ref. ${ }^{24}$ used $\left\langle Z_{S}\right\rangle$ instead of $Z$ in the definition of CLF potential, eq. 32, which explains the paradoxical result $v=1$. Substituting $Z$ instead leads to the expected result $v=3 / 2$.

We note that the result eq. 35 shows that the effective number of entanglements as defined in the tube model is smaller than the average number of slip-links $Z_{s}$. This conclusion is valid for a broader class of models such as slip-springs ${ }^{15}$ and Naples. ${ }^{25}$ Indeed, the reason for $Z<\left\langle Z_{S}\right\rangle$ is the broad distribution of the tube segment lengths, shared by the aforementioned models. For the case of equal size segments we get $Z=\left\langle Z_{s}\right\rangle$. This probably explains why Masubuchi et. al. report smaller modulus than expected from the number of entanglements, and why the slip-springs simulations with $N_{e}=4$ agree with the tube model which uses $N_{e} \approx 6 .^{16}$

\section{Appendix B: Exact mean-square monomer displacement for the freely-}

\section{jointed tube model}

In this Appendix we derive an exact expression for the monomer MSD for the particular version of the tube model, where tube is assumed to be made of segments of equal length $a$, and the directions of different tube segments are uncorrelated. We will call this model the freely-jointed tube model. We note that the validity of eq. 13 relies on eq. 9, which is only correct for large curvilinear distances $|\Delta s|$. However, for the freelyjointed tube model one can derive an exact expression for the distance between two points $s_{1}$ and $s_{2}$ of the tube separated by the curvilinear distance $\Delta s=s_{2}-s_{1}$. It will obviously depend on the position of the first segment along the tube $s_{1}$. Define the variable $x=a \times \operatorname{ceil}\left(s_{1} / a\right)-s_{1}$ as the distance between the first point and the next tube vertex (where ceil $(x)$ is the smallest integer which is larger than $x$ ). It is then easy to show that

$$
\left\langle\Delta r^{2}\right\rangle= \begin{cases}\Delta s^{2}, & \Delta s \leq x \\ x^{2}+n a^{2}+(\Delta s-n a-x)^{2}, & \Delta s>x\end{cases}
$$


where $n=$ floor $((\Delta s-x) / a)$ is the number of full tube segments between $s_{1}$ and $s_{2}$, and floor $(x)$ is the largest integer which is smaller than $x$. Here the angular brackets stand for averaging over different tube conformations.

This can now be averaged over different positions along the tube $x$

$$
\overline{\left\langle\Delta r^{2}\right\rangle}=\frac{1}{a} \int_{0}^{a}\left\langle\Delta r^{2}\right\rangle d x=\left\{\begin{array}{cc}
\Delta s^{2}\left(1-\frac{\Delta s}{3 a}\right), & s \leq a \\
a \Delta s-\frac{a^{2}}{3}, & s>a
\end{array}\right.
$$

This equation should replace eq. 9 for the freely-jointed tube model. As expected, they agree perfectly for large $\Delta s$. Substituting eq. 36 into eq. 11 , we get the exact result

$$
g_{1, \text { mid }}\left(g_{s}\right)=\left(\frac{a^{2}}{3}+g_{s}\right) \operatorname{erf}\left(\frac{a}{\sqrt{2 g_{s}}}\right)+\frac{a}{3} \sqrt{\frac{2 g_{s}}{\pi}} e^{-\frac{a^{2}}{2 g_{s}}}\left(1+2 g_{s} / a^{2}\right)-\frac{2}{3 a} \sqrt{\frac{2}{\pi}} g_{s}^{3 / 2}-\frac{a^{2}}{3}
$$

The series expansion for large $g_{s}(t)$ is then

$$
g_{1, \operatorname{mid}}(t) \approx a \sqrt{\frac{2}{\pi} g_{s}(t)}-\frac{a^{2}}{3}+\frac{a^{3}}{12} \sqrt{\frac{2}{\pi g_{s}(t)}}+O\left(\frac{a^{5}}{g_{s}^{3 / 2}(t)}\right)
$$

The first term corresponds to eq. 13 as stated in the main text: it does not depend on particular details of the tube statistics. The important result is the next order correction term $-a^{2} / 3$, which is negative, meaning that the tube model results should be smaller than the simple power law prediction. The validity of this term is illustrated by the dashed line in Fig. 17(a).

\section{References}

(1) McLeish, T. C. B. Adv. Phys. 2002, 51, 1379-1527.

(2) Bent, J.; Hutchings, L. R.; Richards, R. W.; Gough, T.; Spares, R.; Coates, P. D.; Grillo, I.; Harlen, O. G.; Read, D. J.; Graham, R. S.; Likhtman, A. E.; Groves, D. J.; Nicholson, T. M.; McLeish, T. C. B. Science 2003, 301, 1691-1695.

(3) Kröger, M. Comput. Phys. Commun. 2005, 168, 209-232.

(4) Tzoumanekas, C.; Theodorou, D. N. Macromolecules 2006, 39, 4592-4604.

(5) Evaraers, R.; Sukumaran, S. K.; Grest, G. S.; Svaneborg, C.; Sivasubramanian, A.; Kremer, K. Science 2004, 823, 823-826. 
(6) Padding, J. T.; Briels, W. J. J. Chem. Phys. 2001, 115, 2846-2859.

(7) Padding, J. T.; Briels, W. J. Twentanglement User's manual; Twente University: Twente, The Netherlands, 2000.

(8) As discussed in Refs. ${ }^{6,7}$ the intersection check fails in the situation where either a bond length is zero or parallel to the obstacle, or if the separation between the bond and line obstacle is zero. In contrast to a normal run, where these situations are unlikely, in the primitive path construction they do occur and we reject any movement in which any of these distances become smaller than $\varepsilon g$, where $\varepsilon$ is of the order of the used floating point precision and $g$ the grid size.

(9) Doi, M.; Edwards, S. F. The theory of polymer dynamics; Oxford University Press: Oxford, United Kingdom, 2007.

(10) Likhtman, A. E.; Ramirez, J. http: / / www. reptate.com, 2009.

(11) Likhtman, A. E. In Polymer science: A comprehesive reference; Matyjaszewski, K., Müller.(eds.), M., Eds.; Elsevier B.V.: Amsterdam, 2012; Vol. 1; pp 133-179.

(12) Likhtman, A. E.; McLeish, T. C. B. Macromolecules 2002, 35, 6332-6343.

(13) Rubinstein, M.; Helfand, E. J. Chem. Phys. 1985, 82, 2477-2843.

(14) Zheligovskaya, E. A.; Ternovskii, F. F.; Khokhlov, A. R. Teoret. Mat. Fiz 1988, 7, 644-653.

(15) Likhtman, A. E. Macromolecules 2005, 38, 6128-6139.

(16) Ramírez, J.; Sukumaran, S. K.; Likhtman, A. E. Macromol. Symp. 2007, 126, 094906,1-11.

(17) Wang, Z.; Larson, R. G. Macromolecules 2008, 41, 4945-4960.

(18) de Gennes, P. G. J. Phys. (France) 1981, 42, 735-740.

(19) Wischnewski, A.; Monkenbusch, M.; Willner, L.; Richter, D.; Likhtman, A. E.; McLeish, T. C. B.; Farago, B. Phys. Rev. Lett. 2002, 88, 058301-1.

(20) Doi, M. J. Polym. Sci. Pol. Lett. 1981, 19, 265-273.

(21) Doi, M.; Kuzuu, N. Y. J. Polym. Sci. Pol. Lett. 1980, 18, 775-780.

(22) Sukumaran, S. K.; Likhtman, A. E. Macromolecules 2009, 42, 4300-4309. 
(23) Nair, D. M.; Schieber, J. D. Macromolecules 2006, 39, 2.

(24) Khaliullin, R. N.; Schieber, J. D. Phys. Rev. Lett. 2008, 100, 188302-1.

(25) Masubuchi, Y.; Takimoto, J.; Koyama, K. J. Chem. Phys. 2001, 115, 4387-4394. 


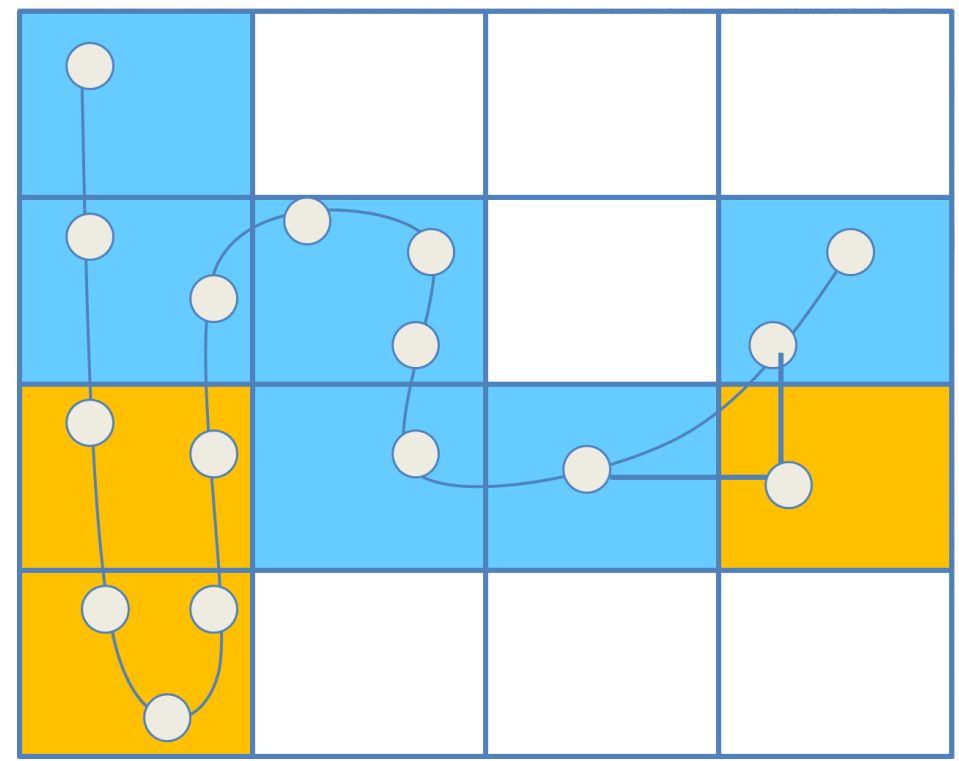

Figure 18: Table of contents graphics

\section{Determination of tube theory parameters using a simple grid model as an example.}

Alexei E. Likhtman, Mohamad S. Talib, Bart Vorselaars, Jorge Ramirez 